\title{
THE
}

9-15-1999

\section{Quantized Systems with Randomly Corrugated Walls and Interfaces}

A. E. Meyerovich

University of Rhode Island, sfo101@uri.edu

A. Stepaniants

University of Rhode Island

Follow this and additional works at: https://digitalcommons.uri.edu/phys_facpubs

Terms of Use

All rights reserved under copyright.

\section{Citation/Publisher Attribution}

Meyerovich, A. E., \& Stepaniants, A. (1999). Quantized Systems with Randomly Corrugated Walls and Interfaces. Phys.Rev. B., 60(12), 9129-9144. doi: 10.1103/PhysRevB.60.9129

Available at: http://dx.doi.org/10.1103/PhysRevB.60.9129

This Article is brought to you for free and open access by the Physics at DigitalCommons@URI. It has been accepted for inclusion in Physics Faculty Publications by an authorized administrator of DigitalCommons@URI. For more information, please contact digitalcommons-group@uri.edu. 


\title{
Quantized systems with randomly corrugated walls and interfaces
}

\author{
A. E. Meyerovich and A. Stepaniants \\ Department of Physics, University of Rhode Island, Kingston, Rhode Island 02881
}

(Received 26 February 1999)

\begin{abstract}
Effect of scattering by random surface inhomogeneities on transport along the walls and localization in ultrathin systems is analyzed. A simple universal surface collision operator is derived outside of the quantum resonance domain. This operator contains all relevant information on statistical and geometrical characteristics of weak roughness and can be used as a general boundary condition on the corrugated surfaces. In effect, the boundary problem for the three-dimensional (3D) transport equation is replaced by the explicit matrix collision operator coupling a set of $2 \mathrm{D}$ transport equations. This operator is applied to a variety of systems including ultrathin films and channels with rough walls, particles adsorbed on or bound to rough substrates, multilayer systems with randomly corrugated interfaces, etc. The main emphasis is on quantization of motion between the walls, though the quasiclassical limit is considered as well. The diffusion and mobility coefficients, localization length, and other parameters are expressed analytically or semianalytically via the intrawall and interwall correlation functions of surface corrugation. [S0163-1829(99)00935-2]
\end{abstract}

\section{INTRODUCTION}

Recent progress in micro- and nanofabrication, multilayer systems, pure materials, vacuum technology, etc., made the study of particle and wave interaction with system boundaries vital for almost all branches of physics. Below, we concentrate on some universal features of wall and interface scattering. More precisely, we consider effects of scattering by random surface corrugation without energy accommodation.

Scattering of particles by random surface inhomogeneities contributes to the randomization of momentum, formation of the mean-free path, quantum interference, and, often, localization. Though this effect of surface scattering looks transparent, it is not easy to express it in terms of geometrical and statistical properties of surface inhomogeneities, especially for quantized systems (see Refs. 1-5 and, for recent references, our preceding publication ${ }^{6}$ ).

At first glance, the effects of scattering by random surface inhomogeneities should not be qualitatively different from scattering by bulk impurities. However, while the basic effects of impurity scattering are described in textbooks, a similar simple account of surface scattering is missing. One of the reasons is technical: the range of impurity interaction is usually short while the corresponding parameter for surface scattering, namely the correlation radius of surface inhomogeneities, can be large. The second reason is more fundamental. It is intuitively clear that the role of surface scattering is higher in ultraquantum small-size systems of longwave particles. In such quantized systems even the impurity scattering is not well understood and is much more complicated than in standard quasiclassical problems.

If one disregards quantum interference and localization, the effect of impurity scattering on quasiclassical threedimensional (3D) transport can be described by the transport equation

$$
\begin{aligned}
\frac{d n(\mathbf{p})}{d t}= & 2 \pi N_{i m p} \int W\left(\mathbf{p}-\mathbf{p}^{\prime}\right)\left[n\left(\mathbf{p}^{\prime}\right)-n(\mathbf{p})\right] \\
& \times \delta\left(\epsilon_{\mathbf{p}}-\epsilon_{\mathbf{p}^{\prime}}\right) \frac{d^{3} p^{\prime}}{(2 \pi)^{3}}
\end{aligned}
$$

where $N_{\text {imp }}$ is the density of impurities, $\epsilon(\mathbf{p})$ is the energy spectrum of particles with the distribution function $n(\mathbf{p})$, and the transition (scattering) probability $W$ between the states $\mathbf{p}$ and $\mathbf{p}^{\prime}$ is proportional to the impurity cross section $\sigma$ (throughout the paper $\hbar=1$ ). This allows to express the relaxation time operator $\hat{\tau}^{-1}$ via the transport cross section $\sigma_{t r}$. In quasi-2D systems with small spacing $L$ between the walls, such as ultrathin films, the motion in $x$ direction perpendicular to the walls is quantized and one deals with a set of $2 \mathrm{D}$ energy minibands $\boldsymbol{\epsilon}_{j}(\mathbf{q})$ instead of the 3D spectrum $\epsilon(\mathbf{p})$. If the motion along the walls remains quasiclassical and the concentration of impurities is large, $N_{i m p}^{1 / 3} L \gg 1$, the transport Eq. (1) can often be rewritten as a set of coupled equations for minibands $\epsilon_{j}(\mathbf{q})$

$$
\begin{aligned}
\frac{d n_{j}(\mathbf{q})}{d t}= & \frac{2 \pi}{L} N_{i m p} \sum_{j^{\prime}} \int W_{j j^{\prime}}\left(\mathbf{q}-\mathbf{q}^{\prime}\right)\left[n_{j^{\prime}}\left(\mathbf{q}^{\prime}\right)-n_{j}(\mathbf{q})\right] \\
& \times \delta\left(\boldsymbol{\epsilon}_{j \mathbf{q}}-\boldsymbol{\epsilon}_{j^{\prime} \mathbf{q}^{\prime}}\right) \frac{d^{2} q^{\prime}}{(2 \pi)^{2}},
\end{aligned}
$$

where the collision integral includes all impurity scattering processes with and without interband transitions $j \leftrightarrow j^{\prime}$. The related relaxation time operator is a matrix, $\widehat{\tau_{j j^{\prime}}^{-1}}$. A similar matrix equation in kinetic theory of gases of particles with discrete internal states is sometimes called the WaldmannSnider transport equation. Since the (weak) localization length is exponentially large in $2 \mathrm{D}$ systems, the quantum interference and localization problem can be approached af- 
ter the "classical" transport problem (2) is solved and the relaxation and diffusion parameters are found.

The form of the collision operators in Eqs. (1) and (2) is universal for bulk impurities and does not depend on the geometry of the system. The main conclusion of this paper is that for a wide range of wall scattering problems one can still use the impuritylike Eq. (2) in which the transition probability $W$ [i.e., the impurity cross-section $\left.\sigma_{j j^{\prime}}\left(\mathbf{q}-\mathbf{q}^{\prime}\right)\right]$ should be simply replaced by the correlation function of surface inhomogeneities $\zeta_{j j^{\prime}}\left(\mathbf{q}-\mathbf{q}^{\prime}\right)$ with trivial constant factors. The form of the resulting transport equation is system independent in the same sense as Eq. (2) is independent of a particular realization of the random impurity system. We solve both the diffusion and localization problems and give a compact expression for the wall-related relaxation time operator $\widehat{\tau_{j j^{\prime}}^{-1}}$. In certain situations, the quantum transport equation is solved analytically. To illustrate the versatility of the results, we present a wide spectrum of applications such as conductivity of ultrathin metal films and channels, multilayer systems, single-particle diffusion of quasiparticles in helium systems, quantum bouncing ball problem for trapped ultracold neutrons or electrons on helium surface, weakly bound states on corrugated substrates, etc.

Our preceding paper ${ }^{6}$ contains a rigorous diagrammatic derivation of the quantum transport equation for quasi-2D systems with weak scattering by random rough walls (or random impurities). The essential difference from the standard Keldysh technique in combination with impurity averaging ${ }^{7}$ is the quantization of motion in $x$ direction perpendicular to the walls resulting in the quantum (matrix) form of the transport equation; the motion along the walls remains quasiclassical. Almost always, this quantum transport equation with surface or impurity scattering reduces to the form (2). The exception is a narrow quantum resonance region in which the gaps between the quantized energy levels $\Omega$ are comparable to the effective corrugation-induced perturbation. Since the energy gaps $\Omega$ increase with decreasing spacing $L$ between the walls as $1 / L^{2}$, this anomalous quantum resonance domain is unavoidable with miniaturization of the system. In the quantum resonance regime, the description of weak impurity and surface scattering becomes almost intractable. In this regime, transport processes are coupled to off-diagonal (mixed) quantum states and cannot be approximated by Eq. (2), which accounts only for the diagonal (pure) states. Outside of this quantum resonance region, the contribution of the off-diagonal mixed states is small and the quantum transport equation acquires the standard Boltzmann-WaldmannSnider form (2). Below we study only the "normal" regime (2) and show that the surface-induced transition probability $W$ is system independent for a wide range of systems and physical problems.

The wall roughness affects physical processes via the correlation (or lack thereof) of classical and quantum multiple scattering by the walls. The magnitude of the effect is a complicated function of four spatial scales-the particle wavelength $\lambda$, distance $L$ between the walls, bulk mean-free path $\mathcal{L}_{b}$, and the correlation radii $R$ of intra and interwall correlations of surface inhomogeneities. Another spatial scale, namely, the amplitude $l$ of the wall corrugation, determines the "strength" of the surface scattering and, without the bulk scattering and outside of the resonance region, enters the results as a simple perturbative factor $l^{2}$ (see below). The main restriction on our results is that $l$ is small,

$$
l \ll L, R \ll \mathcal{L}_{b},
$$

while the wavelength $\lambda$ remains arbitrary. This restriction is not overly severe. [For example, the only nontrivial transport problem for narrow channels is that for slight roughness. If the roughness is strong, the particles are dephased by each wall collision, and the transport problem becomes trivial with the mean-free path $\mathcal{L} \sim L]$. Two additional conditions that ensure the quasiclassical motion along the walls and the absence of the quantum resonance are also not very restrictive. 6

The results below are obtained for quasi-2D systems with impenetrable external walls and/or transparent interlayer boundaries in multilayer systems. Since the localization length in weakly inhomogeneous 2D systems is exponentially large, ${ }^{8-13}$ one can start from "usual'" transport and diffusion and study the localization effects after or in the frame of the diffusion problem. ${ }^{14-16}$ The diffusion results, with minor modifications, can be extended to quantized narrow quasi-1D channels as long as we do not consider localization effects, which are much stronger in quasi-1D channels than in quasi-2D films.

\section{CORRUGATION-INDUCED RELAXATION TIME OPERATOR}

\section{A. Systems and problems}

The aim of this paper is to describe the effect of scattering by random surface inhomogeneities on physical processes in ballistic systems with random rough surfaces. The results describe the following classes of problems:

- diffusion flows along random rough walls in thin films, wires, layers, quantum wells, waveguides, etc.;

- quantum and classical bouncing-ball problems with a random rough wall and an arbitrary holding potential $U(x)$, which returns the particle (the "bouncing ball") back to the wall;

- motion and localization of adsorbed or weakly bound particles on rough substrates;

- localization of particles in rough channels;

- transport and localization in multilayer systems with rough transparent interfaces between the layers.

The confinement in the $x$ direction perpendicular to the walls is necessary to ensure the repeated scattering by the walls. This confined motion can be quantized; the motion along the walls is quasiclassical. The particles can have arbitrary spectrum $\boldsymbol{\epsilon}(\mathbf{p})=\boldsymbol{\epsilon}(-\mathbf{p})$, such as $p^{2} / 2 m$, "relativistic', spectrum $c p$, spectra with gaps, etc. Specific physical examples and experimental applications are discussed in Sec. III. Geometrically, the systems can be split into four groups:

- systems with a single random rough wall $x$ $=\xi(y, z), \quad\langle\xi\rangle=0$; the particles are bound to it by some attractive potential $U(x), \quad U(x \rightarrow \infty)=0 ; \quad U=\infty$ on the wall;

- single-wall systems, $x=\xi(y, z)$, with particles confined near the wall by a holding potential, such as gravity or electric field, with $U(x \rightarrow \infty)=\infty$; 
- system with two rough external walls, $x= \pm L / 2$ $\mp \xi_{1,2}(y, z), \quad\left\langle\xi_{1,2}\right\rangle=0$, with an arbitrary potential $U(x)$ in between; $U=\infty$ on the walls;

- multilayer systems with corrugated transparent interfaces between the layers, $x=x_{\alpha}+\xi_{\alpha}(y, z)$, and an arbitrary bulk potential $U(x)$ in between (the potential changes abruptly by $[U]_{\alpha}$ on the interface); the confinement in $x$ direction is ensured either by impenetrable external walls or by a holding potential.

Theoretical description of systems with random boundaries is often hindered by a common difficulty. It can be illustrated in the calculation of the corrugation-induced correction to the matrix element $\left\langle\psi_{1}|\hat{H}| \psi_{2}\right\rangle$ for particles with the Hamiltonian $\hat{H}=\hat{H}_{0}+\hat{V}$ and the orthonormalized wave functions $\psi=\psi^{(0)}+\delta \psi\left(\hat{H}_{0}\right.$ and $\psi^{(0)}$ describe the same system but with flat walls; $\hat{V}$ and $\delta \psi$ are the corrugationinduced changes),

$$
\delta\left\langle\psi_{1}|\hat{H}| \psi_{2}\right\rangle=\left\langle\psi_{1}^{(0)}|\hat{V}| \psi_{2}^{(0)}\right\rangle+\left(E_{1}-E_{2}\right)\left\langle\delta \psi_{1} \mid \psi_{2}^{(0)}\right\rangle .
$$

The wave functions $\psi^{(0)}$ for the flat walls are known and the first term in Eq. (4) is the same as in all perturbative schemes. Analytical or computational calculations of the corrugation-induced $\delta \psi$ require a set of wave functions that can be used as a basis. The use of the basis set assumes that all wave functions are defined in the same space. For randomly corrugated walls, especially with the boundary condition $\psi=0$, the domain of existence of the wave functions is not the same as for the flat-geometry functions $\psi^{(0)}$ and the proper basis set cannot be introduced explicitly. Thus, $\delta \psi$ cannot be easily calculated using the functions $\psi^{(0)}$ as an expansion basis. As a result, the second term in Eq. (4) is much more complicated than the first. This issue arises in almost any theory of systems with random rough walls.

The energy factor in this second term indicates that this term does not contribute to the Waldmann-Snider Eq. (2). As it is shown in Ref. 6, the contribution of this term is large only in the resonance situations when Eq. (2) breaks down. In this paper we do not consider the quantum resonance domain and assume that Eq. (2) is valid. Then the only issue is the calculation of the scattering probability $W_{j j}$, in Eq. (2), i.e., of the matrix elements $\left\langle\psi_{j}^{(0)}|\hat{V}| \psi_{j^{\prime}}^{(0)}\right\rangle$ of the corrugationinduced perturbation $\hat{V}$. The real question is, of course, how to define this "corrugation-induced perturbation $\hat{V}$ ', mathematically. After that, the calculation of the matrix elements becomes straightforward.

\section{B. Theoretical approaches}

There are numerous theoretical approaches to scattering by surface corrugation. We will mention only those that are best suited for transport and dissipative phenomena and ignore the ones aimed at description of wave fronts, diffraction patterns, spectral shifts, mean field corrections, etc. These approaches, though not equally convenient and general, lead to identical results outside of the quantum resonance region. Inside the quantum resonance region, the cumbersome calculations are system specific, while most of the existing approaches fail.

\section{S-matrix approach}

One of the options is to start from the single-wall $S$ matrix. ${ }^{5,17}$ Because of the interwall interference, the two-wall scattering $S$ matrix does not factorize into a product of two single-wall $S$ matrices, and the exact expressions for the single-wall $S$ matrix are not sufficient for solving the multiwall problems in the ultraquantum regime. This approach has not been used beyond the simplest two-wall system without interwall interference. ${ }^{18}$

\section{Adiabatic approach}

Kawabata's "adiabatic" approximation for the wave function ${ }^{19}$ can circumvent the difficulties caused by the lack of explicit basis wave functions in systems with randomly corrugated boundaries. Suppose one deals with a flow cannel with two random walls,

$$
x= \pm L / 2 \mp \xi_{1,2}(y, z) .
$$

If the change of the wave function along the walls is slow, one can start from the "adiabatic" wave function

$$
\Psi \sim \exp \left(i q_{y} y+i q_{z} z\right) \sin \left[\pi j \frac{x+L / 2-\xi_{2}(y, z)}{L-\xi_{1}(y, z)-\xi_{2}(y, z)}\right] .
$$

This wave function assumes a slow variation of the wall shape along the channel, $q R \gg j, j l / L(R$ is the "size" of inhomogeneities, i.e., the correlation radius of surface corrugation). This condition differs from the perturbative condition (3) and is sufficient for the calculation of the matrix elements and the reflection coefficient. However, the transport calculations use the perturbative Boltzmann equation, which requires an extra condition of the smallness of the wall corrugation. As a result, the transport restrictions in Ref. 19 are equivalent to supplementing of Eq. (3), $l \ll R, L$, by a strong extra condition $q R \gg S(S$ is the number of occupied and/or accessible minibands). Taken together, these restrictions are stronger than the ones used in other calculations. On the positive side, the adiabatic method, when applicable, makes the physics transparent. The adiabatic method fails in the quantum resonance regime.

\section{Mapping transformation}

One of two approaches used in this paper is the exact mapping of the problem with the corrugated boundaries onto an equivalent problem with flat boundaries and distorted bulk. This approach to transport was suggested first by Tesanovic et al. ${ }^{20}$ and Trivedi and Ashcroft ${ }^{21}$ without an explicit expression for the coordinate transformation. Independently, one of the authors and S. Stepaniants ${ }^{22-24,6}$ and, later, Bratkovsky and Rashkeev ${ }^{25}$ introduced the explicit Migdallike mapping transformation for transport and localization calculations. In a different context, the mapping transformation approach to systems with nonuniform walls has been used for electromagnetic and acoustic wave scattering, diffraction patterns, wave guides, etc. for many years (see Refs. 2,4,8,9 and 26-34 and references therein).

This method provides an unambiguous definition of the corrugation-induced perturbation $\hat{V}$. For a single random rough wall, $x=\xi(y, z)$, the flattening transformation is a simple coordinate shift, 


$$
X=x-\xi(y, z) .
$$

The conjugate momentum transformation,

$$
\hat{p}_{x}=\hat{P}_{x}, \quad \hat{p}_{y, z}=\hat{P}_{y, z}-\xi_{y, z}^{\prime} \hat{P}_{x},
$$

transforms the Hamiltonian $\hat{H}_{0}(\mathbf{p}, \mathbf{r})$ into $\hat{H}_{0}(\mathbf{P}, \mathbf{R})$ $+\hat{V}(\mathbf{P}, \mathbf{R},\{\boldsymbol{\xi}\})$ with a random bulk part $\hat{V}$ that depends on the wall corrugation $\xi(y, z)$. For example, the quadratic Hamiltonian $\hat{H}_{0}=p^{2} / 2 m$ is transformed into

$$
\hat{H}_{0}(\mathbf{p})=\hat{H}_{0}(\mathbf{P})+\hat{V}, \hat{H}_{0}(\mathbf{P})=P^{2} / 2 m, \quad \hat{V}=-\left(\xi_{y}^{\prime}+\xi_{z}^{\prime}\right) \hat{P}_{x} / m
$$

(the distortion operator $\hat{V}$ is linearized in displacement $\xi$ ).

Two walls (5) with the average spacing $L$ can be flattened simultaneously by stretching the film,

$$
X=\frac{x+\xi_{1} / 2-\xi_{2} / 2}{1-\xi_{1} / L-\xi_{2} / L},
$$

while the coordinates in the plane of the wall may be left unchanged,

$$
Y=y, \quad Z=z \text {. }
$$

The conjugate momentum transformation identifies the effective random bulk distortion $\hat{V}\left\{\xi_{1,2}\right\}$. In the case of quadratic Hamiltonian, ${ }^{22-24}$

$$
\begin{gathered}
\hat{H}=\frac{P^{2}}{2 m}+\hat{V}, \quad \hat{V}=\hat{V}_{x}+\hat{V}_{y}+\hat{V}_{z}, \quad \hat{V}_{x}=\frac{\xi_{+}}{m L} \hat{P}_{x}^{2}, \\
\hat{V}_{y, z}=\frac{1}{2 m}\left[\left(\frac{X}{L} \xi_{+y, z}^{\prime}-\frac{1}{2} \xi_{-y, z}^{\prime}\right) \hat{P}_{x} \hat{P}_{y, z}\right. \\
\left.+\hat{P}_{y, z}\left(\frac{X}{L} \xi_{+y, z}^{\prime}-\frac{1}{2} \xi_{-y, z}^{\prime}\right) \hat{P}_{x}\right], \\
\xi_{ \pm}=\xi_{2} \pm \xi_{1},
\end{gathered}
$$

and the problem with the corrugated walls is mapped onto the equivalent bulk problem with flat walls, $X=\mp L / 2$, and randomly distorted bulk, $\hat{V}$. Similar mapping transformation can be used for cylindrical geometry. ${ }^{25}$ If the particle spectrum $\epsilon(\mathbf{p})$ is nonquadratic, the mapping transformation (10) and (11) leads to a more complicated, but still treatable, expression for the effective bulk distortion $\hat{V}$. If the potential field $U(x)$ between the walls is not uniform, the distortion $\hat{V}$ in Eq. (12) should be supplemented by

$$
\frac{\partial U}{\partial X}(x-X) \approx \frac{1}{2} \frac{\partial U}{\partial X}\left[\xi_{2}(1-2 X / L)-\xi_{1}(1+2 X / L)\right] .
$$

One should be cautious when using the mapping transformation in the form (10) and (11): the Jacobian $J$ of this transformation $J \neq 1$. The standard diagrammatic or perturbative techniques implicitly assume that the Jacobian $J=1$. To avoid mistakes, one can restore the volume to $J=1$ by supplementing the transformation (10) by an additional stretching the system in $y, z$ directions instead of Eq. (11). This has been done in Ref. 6. The result shows that the corrections to $\hat{V}$ (12) related to this additional transformation in $y, z$ plane are small and can be disregarded outside of the anomalous quantum resonance region $\Omega \hat{\tau} \sim 1$. Thus, in nonresonance calculations, one can leave the coordinates $y, z$ unchanged and disregard the Jacobian-generated terms in the integrals. By leaving these coordinates unchanged, one can, with the same accuracy, extend the two-wall mapping transformation to the multilayer geometry by applying the coordinate transformations (10) and (11) to each layer independently. Inside the resonance region, this cannot be done since the forced change of the $y, z$ coordinates makes the momenta in each layer different from each other.

The calculation of the matrix elements of the perturbation (9), (12), and (13) is straightforward. Though the mapping transformation and the intermediate expressions for the matrix elements are different for each geometry, the final expression for the transition probabilities $W_{j j^{\prime}}\left(\mathbf{q}, \mathbf{q}^{\prime}\right)$ with $\epsilon_{j}(\mathbf{q})=\epsilon_{j^{\prime}}\left(\mathbf{q}^{\prime}\right)$ for the transport Eq. (2) is not systemspecific with the exception of the quantum resonance regime.

The mapping transformation approach seems indispensable because of its consistency and known accuracy on each step of the calculation. At present, this is the only viable approach for calculations in the anomalous quantum resonance regime.

\section{Direct perturbation calculation}

The simplest approach is to calculate directly the matrix elements of the potential using the wave functions for flat geometry as has been suggested by Fishman and Calecki ${ }^{35}$ for two-wall systems without interwall correlation of inhomogeneities (see also Ref. 36).

In this subsection, this approach is extended to particles with an arbitrary spectrum $\epsilon(\mathbf{p})$, multilayer systems with corrugated external and interlayer walls, and to systems with possible interwall interference and with a nonuniform potential between the walls. The transparent interlayer walls separate different layers of a multilayer system from each other and are characterized by finite steplike jumps $[U]_{\alpha}$ of potential. The potential becomes infinite on external impenetrable walls.

In this approach, the walls are replaced by some infinitely narrow potential $U(x)$. If the straight wall $x=x_{\alpha}$ is described by $U(x)$, the corrugated wall $x=x_{\alpha}+\xi_{\alpha}(y, z)$ is described by $U\left(x+\xi_{\alpha}\right)=U(x)+\hat{V}, \quad \hat{V}=\xi_{\alpha} \partial U / \partial x$. On internal interfaces, $\partial U / \partial x=[U]_{\alpha} \delta\left(x-x_{\alpha}\right)$ and the matrix elements $V_{i k}$ of the "perturbation" $\hat{V}$ are

$$
V_{i k}^{(\alpha)} \equiv \int \exp \left[i \mathbf{s} \cdot\left(\mathbf{q}-\mathbf{q}^{\prime}\right)\right] \xi_{\alpha}(\mathbf{s}) \Psi_{i}[U]_{\alpha} \delta\left(x-x_{\alpha}\right) \Psi_{k} d x d \mathbf{s}
$$

$$
=\xi_{\alpha}\left(\mathbf{q}-\mathbf{q}^{\prime}\right)[U]_{\alpha} \Psi_{i}\left(x_{\alpha}\right) \Psi_{k}\left(x_{\alpha}\right),
$$

where $\mathbf{s}$ is the coordinate in the plane $y, z, \quad \mathbf{q}$ is the conjugate momentum, and the unperturbed wave functions for the flat geometry $\Psi_{i}$ are chosen real.

On external walls, the potential $U$ becomes infinite and should be excluded from the integrals 


$$
\begin{aligned}
(\partial U / \partial x)_{i k} & =\int \Psi_{i}(\partial U / \partial x) \Psi_{k} d x \\
& =-\int U\left(\Psi_{i}^{\prime} \Psi_{k}+\Psi_{i} \Psi_{k}^{\prime}\right) d x,
\end{aligned}
$$

using the Schrodinger equation for the motion in $x$ direction,

$$
\left(\hat{p}_{x}^{2} / 2 m+U\right) \Psi_{i}=\epsilon_{i} \Psi_{i} .
$$

Then

$$
\begin{aligned}
-\int & U\left(\Psi_{i}^{\prime} \Psi_{k}+\Psi_{i} \Psi_{k}^{\prime}\right) d x \\
= & \frac{1}{2 m} \int\left[\Psi_{i}^{\prime} \hat{p}_{x}^{2} \Psi_{k}+\Psi_{k}^{\prime} \hat{p}_{x}^{2} \Psi_{i}\right] d x \\
& -\epsilon_{k} \int \Psi_{i}^{\prime} \Psi_{k} d x-\epsilon_{i} \int \Psi_{i} \Psi_{k}^{\prime} d x,
\end{aligned}
$$

and the integration by parts yields

$$
\begin{aligned}
\left(\frac{\partial U}{\partial x}\right)_{i k}= & \left(\epsilon_{i}-\epsilon_{k}\right) \int \Psi_{i}^{\prime} \Psi_{k} d x \\
& -\frac{1}{2 m} \int\left[\Psi_{i}^{\prime} \frac{\partial}{\partial x} \Psi_{k}^{\prime}+\Psi_{k}^{\prime} \frac{\partial}{\partial x} \Psi_{i}^{\prime}\right] d x \\
= & \left(\epsilon_{i}-\epsilon_{k}\right) \int \Psi_{i}^{\prime} \Psi_{k} d x-\frac{1}{2 m} \Psi_{i}^{\prime}\left(x_{\alpha}\right) \Psi_{k}^{\prime}\left(x_{\alpha}\right) .
\end{aligned}
$$

Since we are interested only in the processes without changes in energy, Eq. (2), i.e., only in the first term in the right-hand side in Eq. (4), the first term in the right-hand side of Eq. (17) should be disregarded. The final expression for the matrix elements of the corrugation-induced "perturbation' of the external wall $(\alpha)$ is [cf. Eq. (14)]

$$
V_{i k}^{(\alpha)}\left(\boldsymbol{\epsilon}_{i \mathbf{q}}=\epsilon_{k \mathbf{q}^{\prime}}\right)=-\frac{1}{2 m} \xi_{\alpha}\left(\mathbf{q}-\mathbf{q}^{\prime}\right) \Psi_{i}^{\prime}\left(x_{\alpha}\right) \Psi_{k}^{\prime}\left(x_{\alpha}\right)
$$

When the particle spectrum is not quadratic, the calculations should be slightly modified. Instead of Eq. (18), one gets

$$
\begin{aligned}
V_{\mathbf{q} j, \mathbf{q}^{\prime} j^{\prime}}\left(\boldsymbol{\epsilon}_{j \mathbf{q}}=\boldsymbol{\epsilon}_{j^{\prime} \mathbf{q}^{\prime}}\right)= & \frac{1}{L} \frac{j j^{\prime}\left(\boldsymbol{\epsilon}_{j^{\prime} \mathbf{q}}-\boldsymbol{\epsilon}_{j \mathbf{q}^{\prime}}\right)}{j^{2}-j^{\prime 2}}\left[\xi_{1}\left(\mathbf{q}^{\prime}-\mathbf{q}\right)\right. \\
& \left.+(-1)^{j+j^{\prime}} \xi_{2}\left(\mathbf{q}^{\prime}-\mathbf{q}\right)\right] .
\end{aligned}
$$

The full matrix element $V_{i k}$ is the sum over $\alpha$ of $V_{i k}^{(\alpha)}$ for all interlayer interfaces (14) and external walls (18) [or (19)].

This approach, by design, disregards the resonance contributions and the second term in the expression (4) for the matrix elements. The accuracy is not always clear; it can be evaluated from the comparison with the more consistent mapping transformation approach. We used both approaches outside of the quantum resonance domain. The transition probabilities $W$ calculated using the matrix elements (14) and (18) turned out to be the same as the ones calculated for $\hat{V}$ (9), (12), and (13) under the condition $E_{i}=E_{k}$ though the matrix elements $V_{i k}$ themselves were not identical.

\section{Scattering probabilities and correlation functions of surface corrugation}

The corrugation-induced bulklike matrix elements $V_{i k}$ (14) and (18) play the same role for boundary scattering as the scattering amplitude for scattering by bulk impurities. The role of the impurity cross section is played by the transition probability which should be averaged over random inhomogeneities $\xi$

$$
W_{j j^{\prime}}\left(\mathbf{q}, \mathbf{q}^{\prime}\right)=\left\langle\left|\sum_{\alpha} V_{j \mathbf{q}, j^{\prime} \mathbf{q}^{\prime}}^{(\alpha)}\right| 2\right\rangle_{\xi} \equiv \sum_{\alpha, \beta} W_{j j^{\prime}}^{(\alpha \beta)}\left(\mathbf{q}, \mathbf{q}^{\prime}\right) .
$$

Since the matrix elements $V_{i k}(14)$ and (18) are linear in $\xi$, the transition probabilities $W(20)$ are quadratic in $\xi$. Their averages are expressed via the correlation functions of surface corrugation defined as

$$
\zeta_{\alpha \beta}(|\mathbf{s}|)=\left\langle\xi_{\alpha}\left(\mathbf{s}_{1}\right) \xi_{\beta}\left(\mathbf{s}_{1}+\mathbf{s}\right)\right\rangle_{\xi} \equiv \int \xi_{\alpha}\left(\mathbf{s}_{1}\right) \xi_{\beta}\left(\mathbf{s}_{1}+\mathbf{s}\right) d \mathbf{s}_{1},
$$

$$
\zeta_{\alpha \beta}(\mathbf{q})=\int d^{2} s e^{i \mathbf{q} \cdot \mathbf{s}} \zeta_{\alpha \beta}(\mathbf{s}) .
$$

The correlation functions $\zeta_{\alpha \alpha}$ describe the intrawall correlations of inhomogeneities, and $\zeta_{\alpha \beta}(\alpha \neq \beta)=\zeta_{\beta \alpha}$ the interwall correlations. Usually, the inhomogeneities on all surfaces are similar, $\zeta_{\alpha \alpha}=\zeta_{\beta \beta}$, but are not correlated between different surfaces, $\zeta_{\alpha \neq \beta}=0$. Occasionally, the corrugations of different boundaries can be correlated with each other, $\zeta_{\alpha \neq \beta} \neq 0 .^{37}$

Experimentally, the correlation function (21) is not necessarily Gaussian. ${ }^{48}$ When possible, we avoid specifying the form of the correlation function and express the results via the angular harmonics of $\zeta_{i k}(\mathbf{s})$. In applications and numerical calculations, when the form of $\zeta_{i k}(\mathbf{s})$ has to be specified, we use the most common Gaussian correlation function

$$
\begin{gathered}
\zeta_{\alpha \beta}(\mathbf{s})=l_{\alpha \beta}^{2} \exp \left(-s^{2} / 2 R_{\alpha \beta}^{2}\right), \\
\zeta_{\alpha \beta}(\mathbf{q})=2 \pi l_{\alpha \beta}^{2} R_{\alpha \beta}^{2} \exp \left(-q^{2} R_{\alpha \beta}^{2} / 2\right) .
\end{gathered}
$$

To avoid the parameter clutter, we assume in numerical applications that all correlation radii $R_{\alpha \beta}$ are the same, while the amplitudes of inhomogeneities may be different with some typical scale $l$

$$
\zeta_{\alpha \beta}(\mathbf{s})=a_{\alpha \beta} l^{2} \exp \left(-s^{2} / 2 R^{2}\right) .
$$

The matrix elements $V_{i k}(14)$ and (18) allow direct calculation of the corrugation-induced transition probabilities $W_{j j^{\prime}}\left(\mathbf{q}, \mathbf{q}^{\prime}\right)$ (20) between the quantum states $(j, \mathbf{q})$ and $\left(j^{\prime}, \mathbf{q}^{\prime}\right)$. For transparent interlayer walls with finite changes in potential $[U]_{\alpha}(14)$, the corrugation-induced transition probabilities (20) are

$$
W_{j j^{\prime}}^{\alpha \beta}=\operatorname{Re}\left[\zeta_{\alpha \beta}\left(\mathbf{q}-\mathbf{q}^{\prime}\right)[U]_{\alpha}\left[U^{*}\right]_{\beta} \Psi_{j \alpha}^{*} \Psi_{j^{\prime} \alpha} \Psi_{j \beta} \Psi_{j^{\prime} \beta}^{*}\right]
$$

where $\Psi_{j \alpha}=\Psi_{j}\left(x_{\alpha}\right)$. Diagonal terms $W_{j j^{\prime}}^{\alpha \alpha}\left(\mathbf{q}-\mathbf{q}^{\prime}\right)$ describe the transitions caused by repeated scattering from one interface $\alpha$. The terms $W^{\alpha \beta}$ with $\alpha \neq \beta$ describe the effect of interwall interference in consecutive scattering from two dif- 
ferent corrugated surfaces. For external impenetrable walls, the probabilities $W(18)$ and (20) are

$$
W_{j j^{\prime}}^{(\alpha \beta)}=\frac{1}{4 m^{2}} \operatorname{Re}\left[\zeta_{\alpha \beta} \Psi_{j \alpha}^{*} \Psi_{j^{\prime} \alpha}^{\prime} \Psi_{j \beta}^{\prime} \Psi_{j^{\prime} \beta}^{*^{\prime}}\right],
$$

and the external wall $(\alpha)$ - interlayer wall $(\beta)$ interference term in the transition probability is

$$
W_{j j^{\prime}}^{(\alpha \beta)}=\frac{-1}{2 m} \operatorname{Re}\left(\zeta_{\alpha \beta}[U]_{\beta} \Psi_{j \alpha}^{*} \Psi_{j^{\prime} \alpha}^{\prime} \Psi_{j \beta} \Psi_{j^{\prime} \beta}^{*}\right) .
$$

Equation (24) for internal interfaces can be used for particles with any spectrum $\epsilon(\mathbf{p})=\epsilon(-\mathbf{p})$, while Eqs. (25) and (26) assume $\epsilon=p^{2} / 2 m$. For particles with an arbitrary spectrum $\epsilon(\mathbf{p})=\epsilon(-\mathbf{p})$ (19) in a homogeneous two-wall system, Eq. (25) should be replaced by

$$
W_{j j^{\prime}}^{(\alpha \beta)}=\frac{\zeta_{\alpha \beta} j^{2} j^{\prime 2}}{L^{2}} \frac{\left[\boldsymbol{\epsilon}_{j \mathbf{q}^{\prime}}-\boldsymbol{\epsilon}_{j^{\prime} \mathbf{q}}\right]^{2}}{\left(j^{2}-j^{\prime} \xi^{2}\right.}\left[\delta_{\alpha \beta}-\left(1-\delta_{\alpha \beta}\right)(-1)^{j+j^{\prime}}\right] .
$$

The most prominent feature of the above scattering probabilities is that these expressions are not system specific and the form of the equations is universal. All system-specific information is hidden, in the form of simple constant factors, in the boundary values of the wave functions (or their derivatives) for ideal flat geometry.

\section{Standard Waldmann-Snider transport equation and the relaxation time}

The transport equation outside of the quantum resonance region $\Omega \tau \sim 1$ has the standard Boltzmann-WaldmannSnider form (2) and is determined by the sum of all corrugation-induced $W^{(\alpha \beta)}$

$$
\frac{d n_{j}}{d t}=2 \pi \sum_{\alpha, \beta, j^{\prime}} \int W_{j j^{\prime}}^{(\alpha \beta)}\left[n_{j^{\prime}}-n_{j}\right] \delta\left(\boldsymbol{\epsilon}_{j \mathbf{q}}-\boldsymbol{\epsilon}_{j^{\prime} \mathbf{q}^{\prime}}\right) \frac{d^{2} q^{\prime}}{(2 \pi)^{2}} .
$$

The integration over $d q^{\prime}$ is done using the $\delta$ function, $\delta\left(\boldsymbol{\epsilon}_{j \mathbf{q}}-\boldsymbol{\epsilon}_{j^{\prime} \mathbf{q}^{\prime}}\right)=\delta\left(q^{\prime}-q_{j j^{\prime}}\right) / \mathbf{v}_{j j^{\prime}}$, where $q_{j j^{\prime}}(q)$ is the solution of the equation $\boldsymbol{\epsilon}_{j^{\prime}}\left(\mathbf{q}_{j j^{\prime}}\right)=\boldsymbol{\epsilon}_{j}(\mathbf{q})$ and $\mathbf{v}_{j j^{\prime}}$ $=\left(\partial \boldsymbol{\epsilon}_{j^{\prime} \mathbf{q}^{\prime}} / \partial q^{\prime}\right)_{q^{\prime}=q_{j j^{\prime}}}$ for isotropic $\boldsymbol{\epsilon}_{j \mathbf{q}}$. As always in the transport theory, the angular integration is eliminated by using the angular harmonics. The currents are given by the first harmonic of the distribution $n_{j}^{(1)} \equiv \nu_{j}$ the equation for which involves only the zeroth and first harmonics ${ }^{(0,1)} W_{j j^{\prime}}\left(q, q_{j j^{\prime \prime}}\right)$ of $W\left(\mathbf{q}-\mathbf{q}_{j j^{\prime}}\right)$ over the angle $\widehat{\mathbf{q q}}_{j j^{\prime}}$,

$$
\begin{gathered}
d \nu_{j}(q) / d t=-\sum_{j^{\prime}} \nu_{j^{\prime}}\left(q_{j j^{\prime}}\right) / \tau_{j j^{\prime}}, \\
\frac{2}{\tau_{j j^{\prime}}}=\sum_{\alpha, \beta, j^{\prime \prime}}\left[\left(\delta_{j j^{\prime}}{ }^{(0)} W_{j j^{\prime \prime}}^{(\alpha \beta)}-\delta_{j^{\prime} j^{\prime \prime}}{ }^{(1)} W_{j j^{\prime}}^{(\alpha \beta)}\right) \frac{q_{j j^{\prime \prime}}}{\mathrm{v}_{j j^{\prime \prime}}}\right] .
\end{gathered}
$$

This collision operator $\widehat{\tau^{-1}}$ can serve, outside of the anomalous quantum resonance regime, as a general boundary condition for a wide range of confined ballistic systems.
In effect, we replaced a boundary problem for the 3D transport equation by the matrix collision operator that couples a set of 2D transport Eqs. (29). This matrix-transport equation and the relaxation time operator closely resemble those for the bulk impurity problem. The angular harmonics of the surface correlation function, $\zeta^{(0)}\left(\left|\mathbf{q}-\mathbf{q}_{j j^{\prime}}\right|\right)-\zeta^{(1)}\left(\left|\mathbf{q}-\mathbf{q}_{j j^{\prime}}\right|\right)$, play the role of the impurity transport cross section, $\sigma_{t r}\left(\left|\mathbf{q}-\mathbf{q}_{j j^{\prime}}\right|\right)=\sigma^{(0)}-\sigma^{(1)}$.

Equations (29) for the distribution functions $\nu_{j}(q)$ are still very complicated. The level of complexity depends on the number of equations $S$ and by the range of $q$ for which this set should be solved. The number of equations is given by the number $S$ of occupied or energetically accessible minibands $\boldsymbol{\epsilon}_{j}(\mathbf{q})$ and can be very large or even infinite. In each equation, the arguments of the distribution functions $\nu_{j}$ and $\nu_{j^{\prime}}, q$, and $q_{j j^{\prime}}(q)$, are different reflecting the integral nature of the collision operator. Sometimes, the number of equations and the number of the relevant values of $q$ become finite. For degenerate fermions, the values of $q$ and $q_{j j^{\prime}}$ in the equation for $\nu_{j}(q)$ are the Fermi momenta $q_{j}$ and $q_{j^{\prime}}$ for minibands $j$ and $j^{\prime}, \quad E_{F}=\epsilon_{j}\left(q_{j}\right)=\epsilon_{j^{\prime}}\left(q_{j^{\prime}}\right)$, while the number $S$ of the occupied minibands $\left(\epsilon_{j}(0) \leqslant E_{F}\right)$ is restricted by the Fermi energy $E_{F}$. For the single-particle problems, the values of $q$ and $q_{j j^{\prime}}$ are the momenta $q_{j}$ and $q_{j}$ of the particle with the overall energy $E$ in the minibands $j$ and $j^{\prime}, \quad E=\epsilon_{j}\left(q_{j}\right)=\epsilon_{j^{\prime}}\left(q_{j^{\prime}}\right)$, while only a finite number of the minibands, $\epsilon_{j}(0) \leqslant E$, are energetically accessible. In other situations, as, for example, for particles with the Boltzmann distribution function, the number of occupied minibands is technically infinite, while the equations should be solved for all values of $q$.

In three important situations the equations decouple from each other, the matrix $\tau_{j j^{\prime}}^{-1}$ becomes diagonal, $\tau_{j j^{\prime}}^{-1}$ $\simeq\left(1 / \tau_{j}\right) \delta_{j j^{\prime}}$, and Eqs. (29) can be solved analytically. This happens when (i) only the first (lowest) quantum state $j$ is occupied or is energetically accessible, $S=j=j^{\prime}=1$; then the set (29) reduces to a single equation

$$
\frac{2}{\tau_{1}}=\sum_{\alpha, \beta}\left[{ }^{(0)} W_{11}^{(\alpha \beta)}-{ }^{(1)} W_{11}^{(\alpha \beta)}\right] \frac{q_{11}}{\mathrm{v}_{11}} ;
$$

(ii) $L \ll R$; the small clearance between the surfaces makes the energy gaps between the minibands $\Omega_{j j^{\prime}} \propto 1 / L^{2}$ large leading to a suppression of the interband transitions in comparison to intraband scattering, $W_{j \neq j^{\prime}} \ll W_{j j}$

$$
\frac{1}{\tau_{j}}=\frac{1}{2} \sum_{\alpha, \beta}\left[{ }^{(0)} W_{j j}^{(\alpha \beta)}-{ }^{(1)} W_{j j}^{(\alpha \beta)}\right] \frac{q_{j j}}{\mathrm{v}_{j j}} ;
$$

(iii) the particle wavelength is large, $q R \ll 1$, and all the transition probabilities $W$ are constant ${ }^{(0)} W \approx 2 W(q=0)$ with the zero first harmonic ${ }^{(1)} W=0$ (quantum reflection, cf. Ref. 35)

$$
\frac{1}{\tau_{j}}=\sum_{\alpha, \beta} \sum_{j^{\prime}} W_{j j^{\prime}}^{(\alpha \beta)}(0) \frac{q_{j j^{\prime}}}{\mathrm{v}_{j j^{\prime}}} .
$$

In all other cases, Eqs. (29) remain coupled and should be solved numerically. The condition (ii) is purely geometrical and depends only on the preparation of the walls and the interwall spacing $L$. The other two situations require that the 
characteristic energy of particles should be small in comparison with the interwall gaps $1 / m L^{2}$ in the case (i) or in comparison with the quantum energy $1 / m R^{2}$ in the case of quantum reflection (iii).

Equations (29)-(32) represent the main general result of the paper. The next section contains the practical applications. The calculations can be done for any surface correlator $\zeta(\mathbf{s})$. We will supplement the results by the commonly used Gaussian correlators (22) with the hypergeometric angular harmonics

$$
{ }^{(0)} \zeta_{i k}-{ }^{(1)} \zeta_{i k}=4 \pi a_{i k} l^{2} R_{1}^{2} F_{1}\left(3 / 2,2,-2 q^{2} R^{2}\right) \text {. }
$$

\section{APPLICATIONS}

\section{A. Application of the general equations}

The form of the general equations for the effective relaxation time (29)-(32) is universal. All system-specific information - the energy spectrum $\epsilon_{j}(\mathbf{q})$ and the unperturbed wave functions $\Psi\left(x_{\alpha}\right)$ is hidden in the expressions for the transition probabilities $W(24)-(27)$.

The practical application of these equations is straightforward. One should start from the Schrodinger equation for the "flat" geometry with uncorrugated surfaces and find the energy spectrum $\boldsymbol{\epsilon}_{j}(\mathbf{q})$ and the values of unperturbed wave functions $\Psi_{j}\left(x_{\alpha}\right)$ on interlayer interfaces and $\Psi_{j}^{\prime}\left(x_{\alpha}\right)$ on external walls. These data, together with the correlation function of surface corrugation, provide the transition probabilities $W_{j j^{\prime}}^{(\alpha \beta)}\left(\mathbf{q}, \mathbf{q}^{\prime}\right)(24)-(27)$ and their angular harmonics ${ }^{(0,1)} W_{j j^{\prime}}^{(\alpha \beta)}$. Then one should solve the transport Eq. (29) or, in simpler situations, use the expressions for the kinetic coefficients via the relaxation time (30)-(32).

Below this procedure is illustrated for diverse physical systems with various geometries.

\section{B. Conductivity and mobility in ultrathin films: degenerate particles}

The most direct application is the calculation of diffusion, conductivity, and mobility coefficients for particles in films and channels with an average clearance $L$ between the corrugated walls in the absence of external field $U(x)$. This was done in detail in our previous $\operatorname{paper}^{6}$ for degenerate fermi-

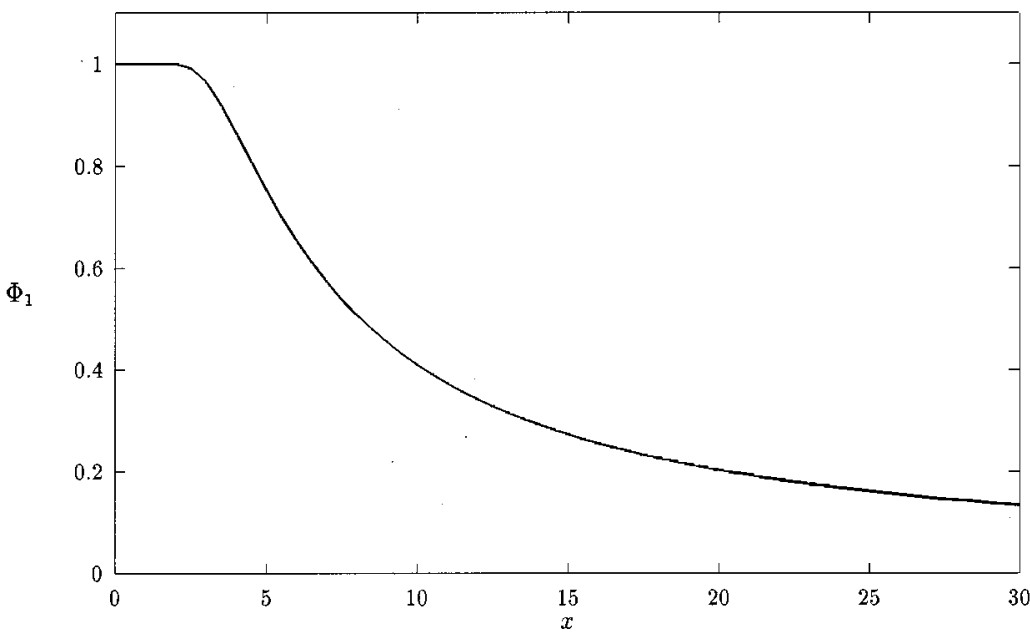

ons. The paper contains the analytical expressions for conductivity $\sigma$, graphs of $\sigma$ in different ranges of $p_{F} R, R / L$, and $S$, the analysis of interwall correlations for this geometry, etc. Though the approach was less general than the one discussed above, the results are the same and should not be repeated.

\section{Conductivity and mobility in ultrathin films: Boltzmann particles}

The transport Eq. (29) for particles with the Boltzmann distribution function, in contrast to the degenerate fermions, involves an infinite number of minibands and should be solved for all values of $q$, and not only for a set of Fermi momenta $q_{j}$.

The convenient representation for the distributions $\nu_{j}$ in Eq. (29) is

$$
\nu_{j}(q)=-\frac{F L^{3}}{\pi^{4} T l^{2}} \exp \left[\frac{\mu-\epsilon_{j \mathbf{q}}}{T}\right] \chi_{j}(q)
$$

( $\mathbf{F}$ is the driving force). Then the conductivity (mobility) acquires the form

$$
\sigma=-\frac{e^{2} L^{3}}{2 \pi^{5} T l^{2} m} \sum_{j} \int q^{2} \exp \left[\frac{\mu-\epsilon_{j \mathbf{q}}}{T}\right] \chi_{j}(q) d q,
$$

while the transport Eq. (29) becomes

$$
q=-\frac{L^{3} m}{\pi^{4} l^{2}} \sum_{j^{\prime}} \chi_{j^{\prime}}\left(q_{j j^{\prime}}\right) / \tau_{j j^{\prime}} .
$$

The relaxation times $\tau_{j j^{\prime}}(q)$ are defined by Eq. (29).

To avoid cumbersome equations, we give the analytical results directly for the Gaussian distribution of inhomogeneities and evaluate the dimensionless conductivity $\Pi(R / L, x)$ defined as

$$
\begin{gathered}
\sigma=\frac{2 e^{2} L^{2}}{\pi^{4} l^{2}} Z \Pi(R / L, x), \\
Z=2 N L^{2} / \pi, \quad x=q_{T} R \equiv \sqrt{2 m T} R .
\end{gathered}
$$

FIG. 1. Function $\Phi_{1}(x)$, Eq. (39). 


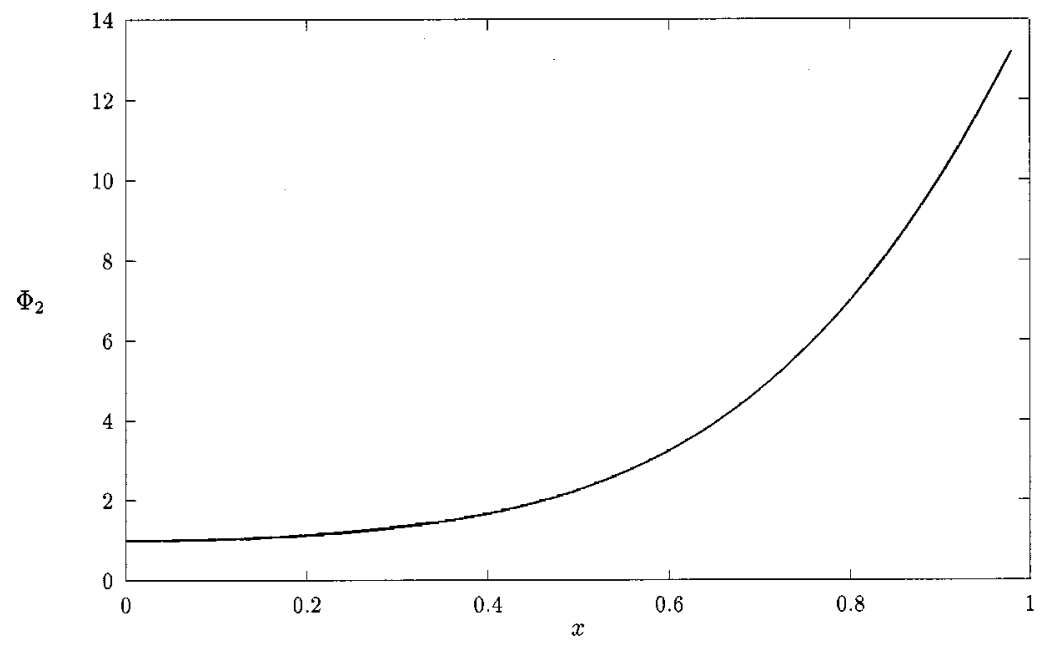

FIG. 2. Function $\Phi_{2}(x)$, Eq. (39).

When the clearance between the walls is small, $q_{T} L \ll 1$, the thermal energy is small in comparison to the interband gaps and only the lowest miniband $\epsilon_{1 \mathbf{q}}$ has a noticeable occupation. Then the dimensionless conductivity (37) becomes

$$
\Pi=\frac{L^{2}}{8 R^{2}} \frac{1}{a_{11}+a_{22}+2 a_{12}} .
$$

Interband transitions are suppressed in comparison with intraband scattering if $R / L \gg 1$ [this is a sufficient condition for $q_{T} L \gtrsim 1$; for small $q_{T} L \ll 1$ one returns to the single-band situation (38)]. Then the dimensionless conductivity $\Pi\left(R / L, q_{T} R\right)$ decouples into the product of two singleparameter functions $\Phi_{1}\left(q_{T} L\right)$ and $\Phi_{2}\left(q_{T} R\right)$

$$
\begin{gathered}
\Pi=\frac{L^{2}}{8 R^{2}} \frac{1}{a_{11}+a_{22}+2 a_{12}} \Phi_{1}\left(q_{T} L\right) \Phi_{2}\left(q_{T} R\right), \\
\Phi_{1}(x)=\frac{\sum j^{-4} \exp \left(-\pi^{2} j^{2} / x^{2}\right)}{\sum \exp \left(-\pi^{2} j^{2} / x^{2}\right)}, \\
\Phi_{2}(x)=\int_{0}^{\infty} \frac{d y y \exp (-y)}{{ }_{1} F_{1}\left(3 / 2 ; 2 ;-2 y x^{2}\right)} .
\end{gathered}
$$

Functions $\Phi_{1}(x)$ and $\Phi_{2}(x)$ are plotted in Figs. 1 and 2.

The third analytical case is the case of long-wave particles $q_{T} R \ll 1$

$$
\begin{gathered}
\Pi=\frac{L^{2}}{8 R^{2}} \frac{1}{a_{11}+a_{22}} \Phi_{3}\left(q_{T} L\right) ; \\
\Phi_{3}(x)=\frac{6 \pi^{2}}{x^{2}}\left[\sum \exp \left(-\frac{\pi^{2} j^{2}}{x^{2}}\right)\right]^{-1} \sum_{j=1}^{\infty} \frac{1}{j^{2}} \sum_{k=j}^{\infty} \frac{Q_{k}^{(j)}(x)-Q_{k+1}^{(j)}(x)}{k(k+1)(2 k+1)\left[1+6(-1)^{j+k} a_{12} /\left(a_{11}+a_{22}\right)(2 k+1)\right]}, \\
Q_{k}^{(j)}(x)=\left(k^{2}-j^{2}+x^{2} / \pi^{2}\right) \exp \left(-\pi^{2} k^{2} / x^{2}\right) .
\end{gathered}
$$

Function $\Phi_{3}(x)$ is plotted in Fig. 3 .

A numerical example for $\Pi$ (37) in an intermediate range of parameters is given in Fig. 4 for two values of the interwall correlation amplitude $a_{12}=0$ (solid curve) and $a_{12}$ $=0.8$ (dotted line). As expected, the gradual filling of minibands for Boltzmann particles results in much smoother curves than for degenerate fermions in Ref. 6 for which the singularities in $\sigma$ reflect abrupt changes in the number of occupied minibands with changing Fermi energy. The effect of interwall correlations is also less dramatic than in the degenerate case.

\section{Single-particle diffusion in thin films and narrow channels}

The single-particle diffusion in channels and films with corrugated walls is similar to the diffusion problem for degenerate fermions. In equilibrium, a particle with energy $E$ can be in any of $S$ accessible minibands $\epsilon_{j}(\mathbf{q})=(1 /$ $2 m)\left[(\pi j / L)^{2}+q^{2}\right]$ for which $\epsilon_{j}(0) \leqslant E$. The equilibrium distribution function $n^{(0)}$ is

$$
n^{(0)}(\mathbf{q})=\sum n_{j}^{(0)}(\mathbf{q})=\frac{\pi}{m S} \sum \delta\left(E-\epsilon_{j}(\mathbf{q})\right)
$$




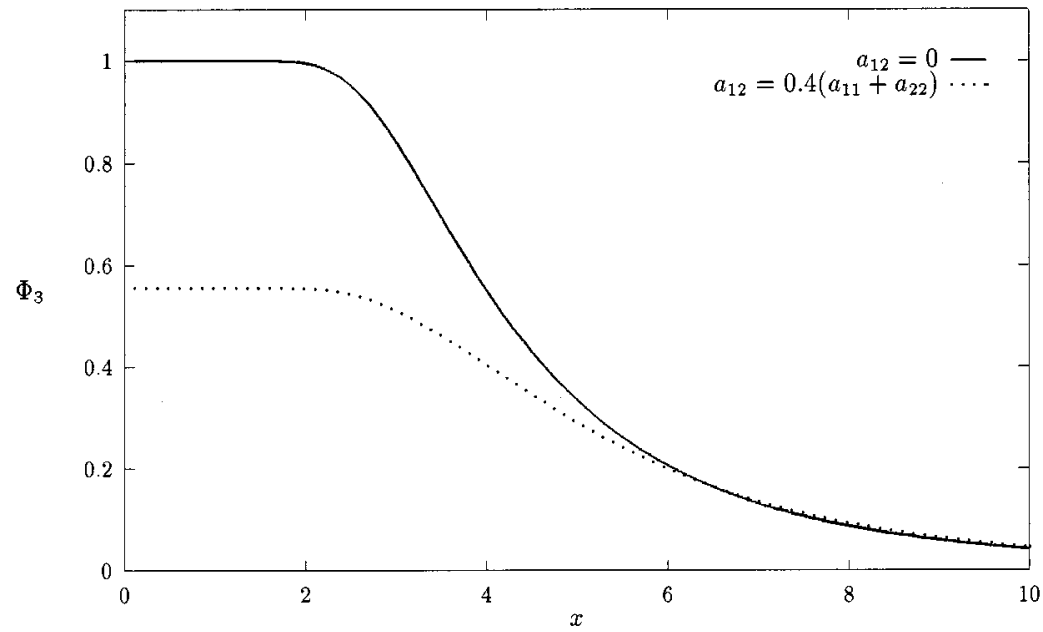

FIG. 3. Function $\Phi_{3}(x)$, Eq. (40), for two values of the interwall correlation amplitude $a_{12}$ $=0$ (solid curve) and $a_{12} /\left(a_{11}+a_{22}\right)=0.4$ (dotted line).

Equations (29) reduce to a set of $S$ coupled linear equations for distributions $\nu_{j}\left(q_{j}\right)$ with the values of momenta $q_{j}$ $=\sqrt{2 m E-(\pi j / L)^{2}}$,

$$
\begin{gathered}
\frac{1}{S m} q_{j} \nabla \rho=-\sum_{j^{\prime}} \nu_{j^{\prime}}\left(q_{j^{\prime}}\right) / \tau_{j j^{\prime}}, \\
D \nabla \rho=-\frac{1}{m} \sum_{j=1}^{S} q_{j} \nu_{j}\left(q_{j}\right),
\end{gathered}
$$

where $\nabla \rho$ is the density gradient that causes the diffusion, $D$ is the single-particle diffusion coefficient, and $\hat{\tau}$ is still defined by Eq. (29). The single-particle mobility coefficient $b$ is related to $D$ by the Einstein equation $D=b E$.

The set of equations Eq. (42) is easily solved numerically. Analytical solutions are available in the same three cases. For low-energy particles [only one band is accessible, Eq. (30)],

$$
\begin{gathered}
D=\frac{2 \pi^{2}}{m}\left(\frac{L}{\pi}\right)^{4} \frac{\tilde{\epsilon}-1}{\Upsilon_{1}^{(0)}-\Upsilon_{1}^{(1)}}, \\
\Upsilon_{j}^{(0,1)} \equiv \Upsilon^{(0,1)}\left(z_{j}\right)={ }^{(0,1)} \zeta_{11}\left(z_{j}\right)+{ }^{(0,1)} \zeta_{22}\left(z_{j}\right)+2^{(0,1)} \zeta_{12}\left(z_{j}\right),
\end{gathered}
$$$$
\tilde{c}=2 m E(L / \pi)^{2}, z_{j}=q_{j} R \text {. For ultrathin channels (31) }
$$

$$
D=\frac{2 \pi^{2}}{m S}\left(\frac{L}{\pi}\right) \sum_{j}^{4} \frac{\tilde{\epsilon}-j^{2}}{j^{4}\left[\Upsilon_{j}^{(0)}-\Upsilon_{j}^{(1)}\right]},
$$

while for a long-wave particle $2 m E R^{2} \ll 1$,

$$
\begin{aligned}
D= & \frac{2 \pi^{2}}{m S}\left(\frac{L}{\pi}\right)^{4} \frac{3}{S(S+1)(2 S+1)\left[\zeta_{11}(0)+\zeta_{22}(0)\right]} \\
& \times \sum_{j=1}^{S} \frac{\tilde{\epsilon}-j^{2}}{j^{2}\left(1+\Xi_{j}\right)}, \\
\Xi_{j}= & \frac{6}{2 S+1} \frac{(-1)^{j+S} \zeta_{12}(0)}{\zeta_{11}(0)+\zeta_{22}(0)}, \quad \zeta_{i k}(0) \equiv \zeta_{i k}(\mathbf{q}=0) .
\end{aligned}
$$

For Gaussian correlations of inhomogeneities, these equations reduce to

$$
\begin{gathered}
D=\frac{\pi}{2 m l^{2} R^{4}}\left(\frac{L}{\pi}\right)^{6} \frac{\Phi\left(z_{1}\right)}{a_{11}+a_{22}+2 a_{12}}, \\
\Phi(x)=\frac{x^{2}}{{ }_{1} F_{1}\left[3 / 2,2,-2 x^{2}\right]},
\end{gathered}
$$

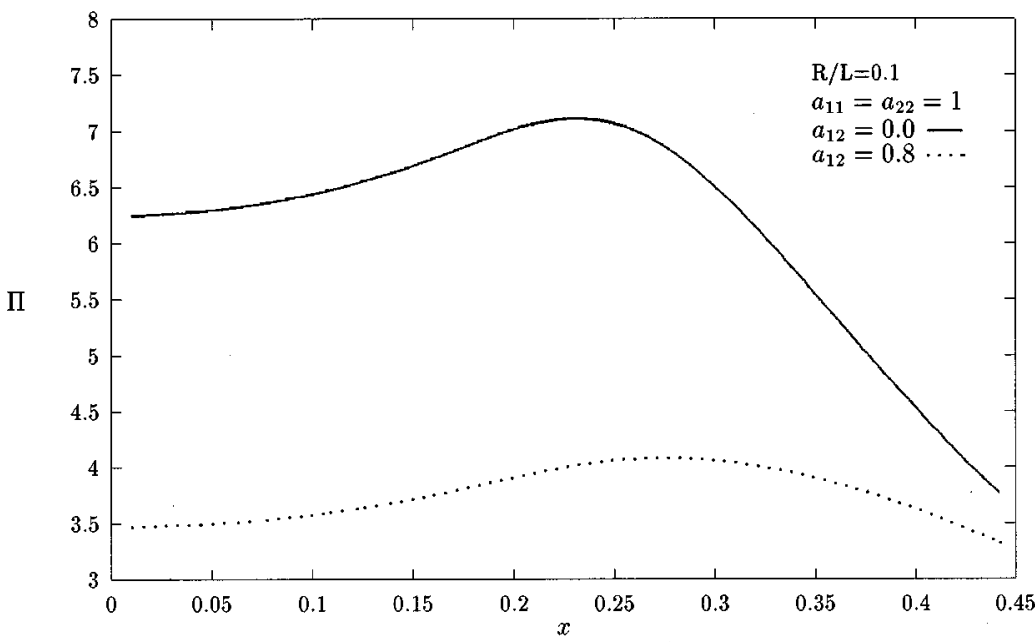

FIG. 4. Function $\Pi(x)$, Eq. (37), for $R / L$ $=0.1, \quad a_{11}=a_{22}=1$, and two values of the interwall correlation amplitude, $a_{12}=0$ (solid curve) and $a_{12}=0.8$ (dotted line). 


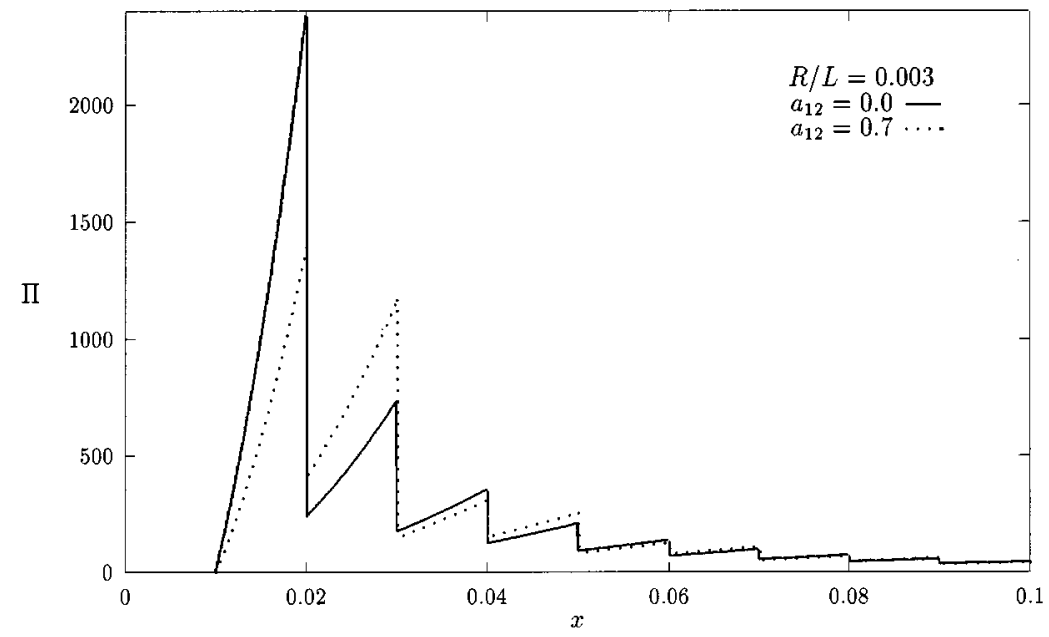

FIG. 5. Function $\Pi(R / L, x), \quad x=R / \lambda$ $=R(2 m E)^{1 / 2}$ for the single-particle diffusion coefficient (49) $D=\left(L^{2} / m l^{2}\right) \Pi(R / L, x)$ at $R / L$ $=0.003, a_{11}=a_{22}=1, a_{12}=0$ (solid line) and $a_{12}=0.7$ (dotted line).

$$
D=\frac{\pi}{2 m S l^{2} R^{4}}\left(\frac{L}{\pi}\right)^{6} \frac{1}{a_{11}+a_{22}+2 a_{12}} \sum_{j} \frac{\Phi\left(z_{j}\right)}{j^{4}},
$$

for ultranarrow channels $R / L \gg 1$, and

$$
\begin{gathered}
D=\frac{\pi}{2 m l^{2} R^{4}}\left(\frac{L}{\pi}\right)^{6} \frac{6}{S^{2}(S+1)(2 S+1)\left(a_{11}+a_{22}\right)} \\
\times \sum_{j=1}^{S} \frac{z_{j}^{2}}{j^{2}\left(1+\Xi_{j}\right)}, \\
\Xi_{j}=\frac{6}{2 S+1} \frac{(-1)^{j+S} a_{12}}{a_{11}+a_{22}},
\end{gathered}
$$

for long-wave particles [here $z_{j}=q_{j} R=(2 \mathrm{mE}$ $\left.\left.-\pi^{2} j^{2} / L^{2}\right)^{1 / 2} R\right]$. An example of the single-particle diffusion coefficient, parameterized as

$$
D=\frac{L^{2}}{m l^{2}} \Pi\left(\frac{R}{L}, R \sqrt{2 m E}\right),
$$

is given in Fig. 5 for $R / L=0.003, a_{11}=a_{22}=1$, and two values of interwall correlations, $a_{12}=0$ (solid line) and $a_{12}$ $=0.7$ (dotted line). The contribution of interwall correlation is an oscillating function of the number $S$ of minibands $\boldsymbol{\epsilon}_{j \mathbf{q}}$ accessible to a particle with energy $E$ and changes from destructive to constructive depending on whether $S$ is even or odd. This is a general feature that can be observed for any energy spectrum at $R \ll \lambda \leqslant L$ for not very large values of $S$. At larger $S$, especially in the quasiclassical regime $S \gg 1$, the contribution of interwall correlations vanishes.

\section{E. Quasiparticles with nonquadratic energy spectrum}

So far, we were discussing the effect of weak surface roughness on particles with quadratic energy spectrum, $\boldsymbol{\epsilon}_{j}(\mathbf{q})=\left[(\pi j / L)^{2}+q^{2}\right] / 2 m$. In this subsection we analyze the single-particle diffusion coefficient for particles with a nonquadratic spectrum such as ballistic solid-state electrons or quasiparticles in low-temperature helium systems with a low probability of inelastic processes. The latter include phonons $\epsilon_{j \mathbf{q}}=c\left[q^{2}+(\pi j / L)^{2}\right]^{1 / 2}, \quad{ }^{3}$ He quasiparticles in He II, and ballistic quasiparticles in low-temperature ${ }^{3} \mathrm{He}$. The scatter- ing by wall inhomogeneities defines the helium flow through capillaries or superleaks at ultralow temperatures and the thermomechanical effect. In superfluid ${ }^{3} \mathrm{He}$, the results are affected by Andreev reflection. To account for these processes, the transport Eq. (29) should be re-written as two coupled sets of equations for quasiparticles and quasiholes.

The form of the transport Eq. (29) formally does not change irrespective of the particle spectrum while the transition probabilities acquire the form (27)

$$
\begin{aligned}
W_{j j^{\prime}} \delta\left(\boldsymbol{\epsilon}_{j^{\prime} \mathbf{q}^{\prime}}-\epsilon_{j \mathbf{q}}\right)= & \frac{j^{2} j^{\prime 2}}{L^{2}} \frac{\left[\boldsymbol{\epsilon}_{j \mathbf{q}^{\prime}}-\boldsymbol{\epsilon}_{j^{\prime} \mathbf{q}}\right]^{2}}{\left(j^{2}-j^{\prime 2}\right)^{2}} \\
& \times\left[\zeta_{11}+\zeta_{22}+2(-1)^{j+j^{\prime}} \zeta_{12}\right] \\
& \times \delta\left(\boldsymbol{\epsilon}_{j^{\prime} \mathbf{q}^{\prime}}-\boldsymbol{\epsilon}_{j \mathbf{q}}\right) .
\end{aligned}
$$

If only one energy miniband is occupied, $E=\epsilon_{1}\left(\mathbf{q}_{1}\right)$ $<\epsilon_{2}(0)$, the single-particle diffusion coefficient (30) is

$$
D(E)=\frac{2 L^{2}}{\Upsilon_{11}^{(0)}-\Upsilon_{11}^{(1)}} \frac{\left(\partial \boldsymbol{\epsilon}_{1 \mathbf{q}_{1}} / \partial q_{1}\right)^{3}}{\left.q_{1}\left(\partial \boldsymbol{\epsilon}_{j \mathbf{q}_{j}} / \partial j\right)^{2}\right|_{j=1}},
$$

where the angular harmonics are defined as

$$
\begin{aligned}
\Upsilon_{j j^{\prime}}^{(0,1)}= & { }^{(0,1)} \zeta_{11}\left(\left|\mathbf{q}_{j}-\mathbf{q}_{j^{\prime}}^{\prime}\right|\right)+{ }^{(0,1)} \zeta_{22}\left(\left|\mathbf{q}_{j}-\mathbf{q}_{j^{\prime}}^{\prime}\right|\right) \\
& +2(-1)^{j+j^{\prime}(0,1)} \zeta_{12}\left(\left|\mathbf{q}_{j}-\mathbf{q}_{j^{\prime}}^{\prime}\right|\right) .
\end{aligned}
$$

In the limit of ultranarrow channels, $L \ll R$, the interband transitions are negligible in comparison with intraband scattering and the diffusion coefficient is (31)

$$
D(E)=\sum_{j=1}^{S} \frac{2 L^{2} / S}{\Upsilon_{j j}^{(0)}-\Upsilon_{j j}^{(1)}} \frac{\left(\partial \boldsymbol{\epsilon}_{j \mathbf{q}_{j}} / \partial q_{j}\right)^{3}}{j^{2} q_{j}\left(\partial \boldsymbol{\epsilon}_{j \mathbf{q}_{j}} / \partial j\right)^{2}} .
$$

In the case of quantum reflection $q_{j} R \ll 1$, all the correlators $\zeta_{i k}\left(\mathbf{q}-\mathbf{q}^{\prime}\right)$ in the kernel of the integral equation can be replaced by the constants $\zeta_{i k}(0)$, and the diffusion coefficient of the quasiparticle with the energy $E=\epsilon_{j \mathbf{q}_{j}}$ is equal to 


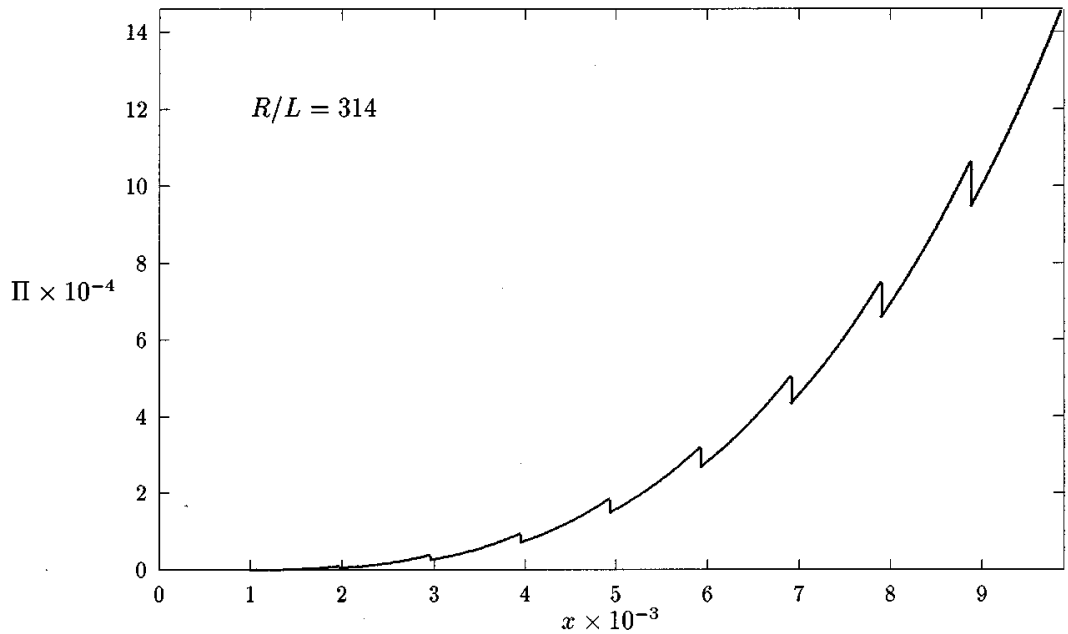

FIG. 6. Function $\Pi(R / L, x), \quad x=R \omega / c$, for the single-phonon diffusion coefficient $D$ $=\left(L^{3} c / l^{2}\right) \Pi(R / L, x)$ at $R / L=314, a_{11}=a_{22}=1$, and $a_{12}=0$.

$$
\begin{aligned}
D(E)= & \frac{L^{2}}{S} \sum_{j=1}^{S}\left(\frac{\partial \boldsymbol{\epsilon}_{j \mathbf{q}_{j}}}{\partial q_{j}}\right)^{2} / \\
& \sum_{j^{\prime}=1}^{S} \frac{q_{j^{\prime}} \Upsilon_{j j^{\prime}}(0) j^{2} j^{\prime 2}\left(\boldsymbol{\epsilon}_{j^{\prime} \mathbf{q}_{j}}-\boldsymbol{\epsilon}_{j \mathbf{q}_{j^{\prime}}}\right)^{2}}{\left(j^{2}-j^{\prime 2}\right)^{2}\left(\partial \boldsymbol{\epsilon}_{j^{\prime} \mathbf{q}_{j^{\prime}}} / \partial q_{j^{\prime}}\right)} .
\end{aligned}
$$

For Gaussian correlations (22), $Y(52)$ is expressed via the hypergeometric function ${ }_{1} F_{1}$

$$
\begin{gathered}
\Upsilon_{j j^{\prime}}(0)=2 \pi\left[a_{11}+a_{22}+2(-1)^{j+j^{\prime}} a_{12}\right] l^{2} R^{2}, \\
\Upsilon_{j j}^{(0)}-\Upsilon_{j j}^{(1)}=4 \pi\left(a_{11}+a_{22}+2 a_{12}\right) l^{2} R_{1}{ }^{2} F_{1}\left(\frac{3}{2}, 2,-2 q_{j}^{2} R^{2}\right) .
\end{gathered}
$$

The frequency dependence of the diffusion coefficient for "phonons" $\boldsymbol{\epsilon}_{j \mathbf{q}}=c\left[q^{2}+(\pi j / L)^{2}\right]^{1 / 2}$ at $R \gg L$,

$$
\begin{gathered}
D=\frac{2}{a_{11}+a_{22}+2 a_{12}} \frac{L^{3} c}{l^{2}} \Pi\left(\frac{R}{L}, x \equiv \frac{R \omega}{c}\right), \\
\Pi=\frac{L^{3} / R^{3}}{4 \pi^{5} x S} \sum_{j=1}^{S} \frac{1}{j^{4}} \frac{z_{j}^{2}}{{ }_{1} F_{1}\left(\frac{3}{2}, 2,-2 z_{j}^{2}\right)}, \\
z_{j}^{2}=x^{2}-\left(\frac{\pi j R}{L}\right)^{2}
\end{gathered}
$$

is illustrated in Fig. 6 for Gaussian correlation of inhomogeneities. Sharp singularities in the curves appear in the points where the number $S$ of minibands $\epsilon_{j \mathbf{q}}$ accessible to the "phonon" with frequency $\omega$ increases by 1 with increasing $\omega$.

\section{F. Multilayer systems with corrugated interlayer walls}

The effect of scattering by corrugated interlayer walls is similar to that for scattering by a corrugated external wall. The simplest illustration with the smallest number of parameters is a two-layer system with flat walls $x=0 ; L$ and a rough interface $x=x_{0}+\xi(y, z)$ between the layers, $0<x_{0}$ $<L$. The potential changes on the interface by $U_{0}$. The transition probability (24) contains the correlation function $\zeta$ of the interface inhomogeneities and the value of the unperturbed wave function $\left|\Psi_{j}\left(x_{0}\right)\right|^{2}$ on the interface,

$$
W_{j j^{\prime}}=\zeta\left(\mathbf{q}-\mathbf{q}^{\prime}\right)\left|U_{0}\right|^{2}\left|\Psi_{j}\left(x_{0}\right)\right|^{2}\left|\Psi_{j^{\prime}}\left(x_{0}\right)\right|^{2} .
$$

The unperturbed spectrum $\boldsymbol{\epsilon}_{j}(\mathbf{q})=e_{j}+q^{2} / 2 m$,

$$
e_{j}=(\pi / L)^{2} \widetilde{e}_{j} / 2 m, \quad U_{0}=(\pi / L)^{2} \kappa / 2 m, \quad x_{0}=L \delta,
$$

and the wave function on the interface $\Psi_{j}\left(x_{0}\right)$ should be found from the Schrodinger equation,

$$
\begin{aligned}
& \frac{1}{\sqrt{\tilde{e}_{j}}} \tan \left[\pi \delta \sqrt{\tilde{e}_{j}}\right]+\frac{1}{\sqrt{\tilde{e}_{j}-\kappa}} \tan \left[\pi(1-\delta) \sqrt{\tilde{e}_{j}-\kappa}\right]=0, \\
& \tilde{e}_{j}>\kappa, \\
& \frac{1}{\sqrt{\tilde{e}_{j}}} \tan \left[\pi \delta \sqrt{\tilde{e}_{j}}\right]+\frac{1}{\sqrt{\kappa-\tilde{e}_{j}}} \tanh \left[\pi(1-\delta) \sqrt{\kappa-\tilde{e}_{j}}\right]=0, \\
& \tilde{e}_{j}<\kappa,
\end{aligned}
$$

as a function of the wall position $\delta=x_{0} / L$ and the potential difference between the layers $\kappa=2 m U_{0}(L / \pi)^{2}$. This information on dimensionless parameters $\tilde{e}_{j}(\delta, \kappa)$ and $\gamma_{j j^{\prime}}(\delta, \kappa)$ $=L^{2}\left|\Psi_{j}\left(x_{0}\right)\right|^{2}\left|\Psi_{j^{\prime}}\left(x_{0}\right)\right|^{2}$ is sufficient to find $W(56)$, solve the transport Eq. (29), and find the conductivity and diffusion coefficients. The kinetic coefficients for a layered system depend not only on the characteristics of the corrugation, $R / L$ and $\lambda / R$ as in the previous sections, but also on the position and "strength" of the interface $\delta$ and $\kappa$.

In some sense, the effect of the transition from a single layer to a multilayer system is similar to the result of change in spectrum discussed in the previous subsection. The main difference between the two-layer metal film with a corrugated interface and a single-layer film with rough walls, apart from the obvious factor $\kappa \gamma$, is the replacement of the quantum numbers $j^{2}$ by $\tilde{e}_{j}$ [for example, the expressions for the Fermi momenta $q_{j}$ in the harmonics ${ }^{(0,1)} \zeta\left(q_{j}\right)$ become $q_{j}^{2} L^{2} / \pi^{2}=\nu-\tilde{e}_{j}$ instead of $\left.\nu-j^{2}\right]$. If only the first miniband $e_{1}+q^{2} / 2 m$ is occupied, then 


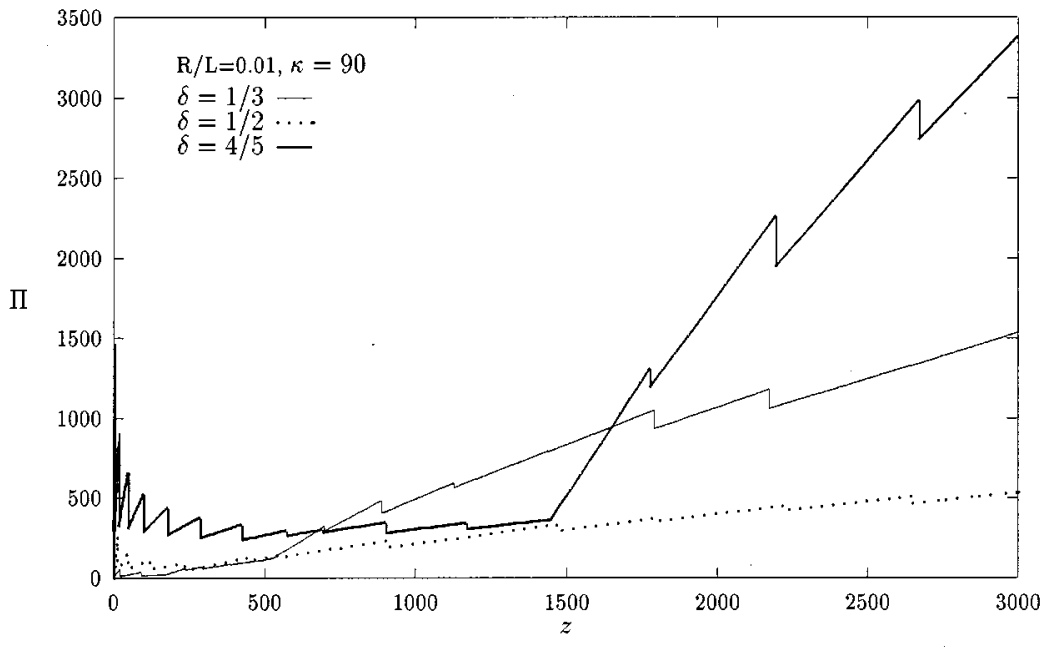

FIG. 7. Function $\Pi(z)$, Eq. (62), for $R / L$ $=0.01$, potential strength $\kappa=90$ for three positions of the interface, $\delta \equiv x_{0} / L=\frac{1}{3} ; \frac{1}{2} ; \frac{4}{5}$.

$$
\sigma=\frac{4 \pi e^{2}}{\gamma_{11} \kappa^{2}}\left(\frac{L}{\pi}\right)^{4} \frac{\nu-\tilde{e}_{1}(\delta, \kappa)}{{ }^{(0)} \zeta\left(q_{1} R\right)-{ }^{(1)} \zeta\left(q_{1} R\right)} .
$$

In ultrathin systems $R / L \gg 1$,

$$
\sigma=\frac{4 \pi e^{2}}{\kappa^{2}}\left(\frac{L}{\pi}\right)^{4} \sum_{j} \frac{1}{\gamma_{j j}} \frac{\nu-\tilde{e}_{j}(\delta, \kappa)}{{ }^{(0)} \zeta\left(q_{j} R\right)-{ }^{(1)} \zeta\left(q_{j} R\right)} .
$$

For the long-wave particles,

$$
\sigma=\frac{2 \pi e^{2}}{\zeta(0) \kappa^{2}}\left(\frac{L}{\pi}\right)^{4} \sum_{j} \frac{\nu-\tilde{e}_{j}}{\sum_{j^{\prime}} \sqrt{\gamma_{j j} \gamma_{j^{\prime} j^{\prime}}}}
$$

Figure 7 presents the function $\Pi(z)$,

$$
\sigma=\frac{2 e^{2} L^{4}}{\pi^{4} l^{2} R^{2} \kappa^{2}} \Pi(z, R / L, \delta, \kappa), \quad z=2 N L^{2} / \pi,
$$

for the interface with the Gaussian correlation of inhomogeneities at $R / L=0.01$ and the potential strength $\kappa$ $=2 m(L / \pi)^{2} U_{0}=90$ for three positions of the interface, $\delta$ $\equiv x_{0} / L=\frac{1}{3} ; \frac{1}{2} ; \frac{4}{5}$. The shape of the curves is less regular and is noticeably different from those for the conductivity of a single-layer film with rough walls.

\section{G. Diffusion of particles bound to rough substrates}

Another application is single-particle diffusion of particles weakly bound to or adsorbed on rough substrates when the size $L$ of the bound state is larger than the amplitude of the surface inhomogeneities, $L \gg l$. Such particles move along the substrate, $\boldsymbol{\epsilon}(\mathbf{q})=\epsilon_{1}+q^{2} / 2 m^{*}$, and experience scattering by its inhomogeneities. We will define the size of the bound state $L$ via the derivative of the unperturbed wave function on the wall,

$$
\frac{\sqrt{2} \pi}{L^{3 / 2}}=\left(\frac{\partial \Psi}{\partial x}\right)_{x=0} .
$$

The coefficients in Eq. (63) are chosen in such a way so that for a channel with two impenetrable walls the value of $L$ in (63) would coincide with the channel width. If there is only one bound state, the expression for the single-particle diffusion coefficient is similar to Eq. (43)

$$
D(E)=\frac{2 \pi^{2}}{m}\left(\frac{L}{\pi}\right)^{6} \frac{q^{2}}{{ }^{(0)} \zeta(q R)-{ }^{(1)} \zeta(q R)},
$$

where $q \equiv\left(2 m E-\epsilon_{1}\right)^{1 / 2}$.

In the case of Gaussian correlation of inhomogeneities, Equation (64) reduces to

$$
D=\frac{\pi}{2 m^{*} R^{4} l^{2}}\left(\frac{L}{\pi}\right)^{6} \Phi(q R),
$$

where the function $\Phi(x)$ is defined by Eq. (46). Possible applications are weakly bound electron states in solids, ${ }^{3} \mathrm{He}$ surface states inside He II, and hydrogen atoms on helium surface. ${ }^{39,40}$ In the last two cases, $\epsilon_{1} \sim 1 \mathrm{~K}, L \sim 12 \AA$, while at $T \sim 1 \mathrm{~K}$ the ripplon corrugation has parameters $R$ $\sim 20 \AA, \quad l \sim 0.8 \AA$, and the coefficient in Eq. (65) is $0.1 \hbar / m$.

\section{H. Bouncing ball problem: electrons on helium surface, neutrons in a gravitational trap, etc.}

A similar class of problems is often referred to as a "bouncing ball', problem. In this problem, a particle bounces repeatedly from a wall after being returned to it by an external field. We are interested in a version of this problem in which the reflection of the quantum particle by the static rough wall $x=\xi(y, z)$ is accompanied by scattering by weak random surface inhomogeneities $\langle\xi\rangle=0$ and leads to the particle diffusion along the wall. Two typical examples are ultracold neutrons in a gravitational trap ${ }^{41}$ and electrons pressed to helium or hydrogen surface by an electric field (see also proceedings ${ }^{42}$ ). In both cases, the holding external fields - $m g x$ and $e E x$, respectively - are linear functions of coordinates making the problems identical.

The transition probability for scattering by the wall inhomogeneities is given by Eq. (25), 


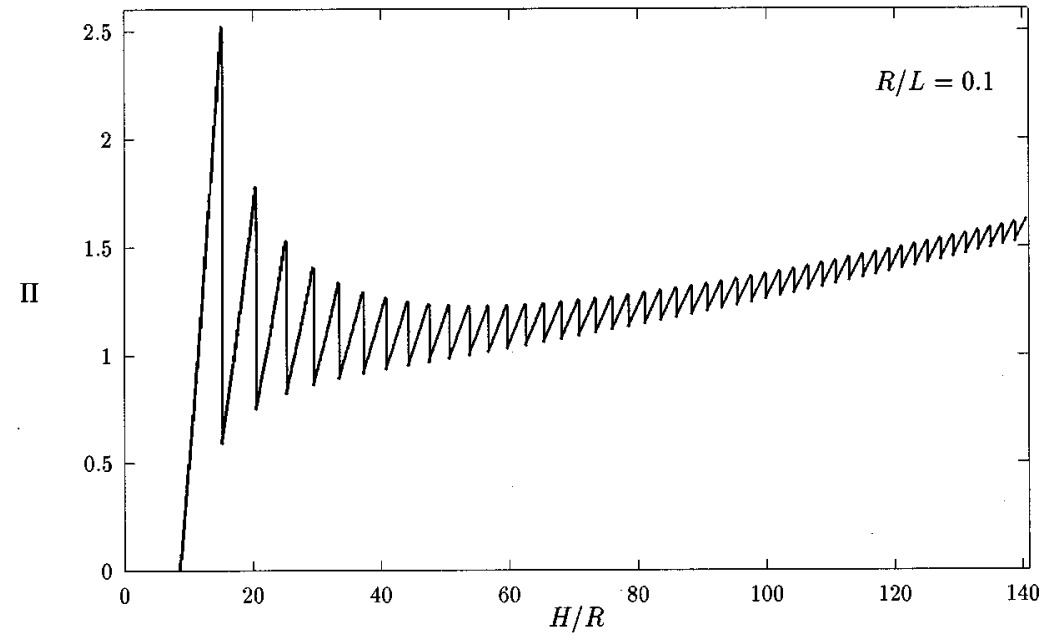

FIG. 8. Function $\Pi(H / R)$, Eq. (74), for $R / L$ $=0.1$.

$$
\begin{aligned}
& W_{j j^{\prime}}\left(\mathbf{q}, \mathbf{q}^{\prime}\right) \delta\left(\boldsymbol{\epsilon}_{j \mathbf{q}}-\boldsymbol{\epsilon}_{j^{\prime} \mathbf{q}^{\prime}}\right) \\
& =\frac{1}{4 m^{2}} \zeta\left(\mathbf{q}^{\prime}-\mathbf{q}\right)\left|\frac{d \Psi_{j}(0)}{d X}\right|^{2}\left|\frac{d \Psi_{j^{\prime}}(0)}{d X}\right|^{2} \delta\left(\boldsymbol{\epsilon}_{j \mathbf{q}}-\boldsymbol{\epsilon}_{j^{\prime} \mathbf{q}^{\prime}}\right)
\end{aligned}
$$

with the energy spectrum $\epsilon_{j \mathbf{q}}=\epsilon_{j}+q^{2} / 2 m$. The unperturbed wave functions for the flat wall and linear holding potential are the Airy functions

$$
\Psi_{j \mathbf{q}}(x)=e^{i \mathbf{q s}} C_{j} \operatorname{Ai}\left(\left(2 m^{2} g\right)^{1 / 3} x-\tilde{\epsilon}_{j}\right),
$$

where $C_{j}$ are the normalization coefficients. The energy eigenvalues $\tilde{\epsilon}_{j}=2^{1 / 3} \pi^{2 / 3} \epsilon_{j} / m g L$ are given by the zeroes of the Airy function, $\operatorname{Ai}\left(-\tilde{\epsilon}_{j}\right)=0 ; \quad \tilde{\epsilon}_{1,2,3, \ldots}=2.34 ; 4.09$; $5.52, \ldots$ The spacial scale is determined by the size of the first, closest to the wall discrete state $L=\left(\pi^{2} / m^{2} g\right)^{1 / 3}$, Eq. (63). Finally, the transition probabilities (66) reduce to

$$
W_{j j^{\prime}}\left(\mathbf{q}, \mathbf{q}^{\prime}\right) \delta\left(\boldsymbol{\epsilon}_{j \mathbf{q}}-\boldsymbol{\epsilon}_{j^{\prime} \mathbf{q}^{\prime}}\right)=m^{2} g^{2} \zeta\left(\mathbf{q}^{\prime}-\mathbf{q}\right) \delta\left(\boldsymbol{\epsilon}_{j \mathbf{q}}-\boldsymbol{\epsilon}_{j^{\prime} \mathbf{q}^{\prime}}\right) .
$$

The transport Eq. (29) with the transition probabilities (68) can be solved analytically in the same three situations. The single-particle diffusion coefficient in the first miniband $\epsilon_{1}(\mathbf{q})$ is

$$
\begin{aligned}
D(E) & =\frac{2 \pi^{2}}{m}\left(\frac{L}{\pi}\right)^{6} \frac{q_{1}^{2}}{{ }^{(0)} \zeta\left(q_{1} R\right)-{ }^{(0)} \zeta\left(q_{1} R\right)} \\
& \rightarrow \frac{\pi}{2 m R^{4} l^{2}}\left(\frac{L}{\pi}\right)^{6} \Phi\left(q_{1} R\right),
\end{aligned}
$$

where the last expression describes Gaussian correlations of wall inhomogeneities; $q_{1}^{2}=2 m\left(E-\epsilon_{1}\right)$, and $\Phi(x)$ is given by Eq. (46). Particles cannot access the second miniband as long as $4.13<\sqrt{2 m E} L<5.45$.

In the case of large interband spacing $L^{2} / R \lambda$ $\sim \sqrt{2 m E} L^{2} / R \ll 1$, the interband transitions are suppressed and the transport Eqs. (29) decouple. Then the diffusion coefficient is

$$
\begin{aligned}
& D\left(\sqrt{2 m E} L^{2} / R \ll 1\right) \\
& =\frac{4}{m^{4} g^{2} S} \sum_{j=1}^{S} \frac{E-\epsilon_{j}}{(0) \zeta\left(2 q_{j} \sin \frac{\theta}{2}\right)-{ }^{(1)} \zeta\left(2 q_{j} \sin \frac{\theta}{2}\right)},
\end{aligned}
$$

where $q_{j}^{2}(E)=2 m\left(E-\epsilon_{j}\right)$. In the case of Gaussian correlations, the diffusion coefficient is

$$
\begin{aligned}
D\left(\sqrt{2 m E} L^{2} / R \ll 1\right) & \\
= & \frac{1}{\pi m^{4} g^{2} R^{2} l^{2} S} \sum_{j=1}^{S} \frac{E-\epsilon_{j}}{{ }_{1} F_{1}\left(3 / 2 ; 2 ;-2 q_{j}^{2} R^{2}\right)} \\
& \rightarrow \frac{5 \pi^{3 / 2} R H^{4}}{8 \sqrt{2} m L^{3} l^{2} S}
\end{aligned}
$$

(the last equation is quasiclassical for a large number of accessible minibands $S \gg 1 ; \quad H=E / m g$ is the maximal jump amplitude).

In the case of the long-wave particles, $\sqrt{2 m E} R=R / \lambda$ $\ll 1$, the solution of Eq. (29) yields

$$
D(R / \lambda \ll 1)=\frac{2}{m^{4} g^{2} S^{2} \zeta(0)} \sum_{j=1}^{S}\left(E-\epsilon_{j}\right) \rightarrow \frac{4}{5 \pi^{2}} \frac{H L^{3}}{m S \zeta(0)}
$$

or, for Gaussian correlations,

$$
D(R / \lambda \ll 1)=\frac{1}{\pi m^{4} g^{2} S^{2} R^{2} l^{2}} \sum_{j=1}^{S}\left(E-\epsilon_{j}\right) \rightarrow \frac{2}{5} \frac{H L^{3}}{\pi^{3} m S R^{2} l^{2}}
$$

[the last expressions in Eqs. (72) and (73) are quasiclassical, $S \gg 1]$.

A numerical example of the diffusion coefficient

$$
D=\frac{2 L^{2}}{m l^{2}} \Pi(R / L, H / R)
$$

is given in Fig. $8(H \equiv E / m g$ is the amplitude of the particle jumps). 


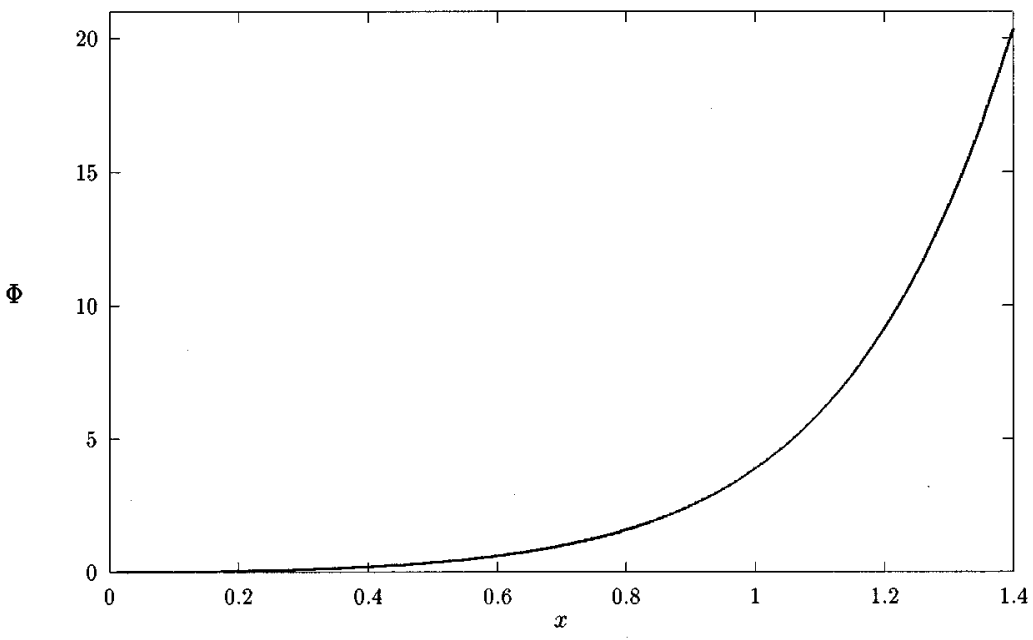

FIG. 9. Function $\Phi(x)$, Eqs. (46) and (76).

One of the most interesting applications is the system of ultracold neutrons ${ }^{41}$ in a gravitational trap with a macroscopically inhomogeneous "floor.' The typical neutron parameters are $L=\left(\pi^{2} / \mathrm{m}^{2} \mathrm{~g}\right)^{1 / 3}=1.58 \times 10^{-3} \mathrm{~cm}, \sqrt{2 m E} R$ $=1.6 \times 10^{3} R \mathrm{~V}$ (here $R$ is measured in $\mathrm{cm}$, and the neutron velocity $\mathrm{v}=\sqrt{2 E / m}$-in $\mathrm{cm} / \mathrm{s})$. At present, the neutrons can be trapped with velocities down to $\mathrm{V}=100 \mathrm{~cm} / \mathrm{s}$ (jump amplitudes $H \sim 5 \mathrm{~cm}){ }^{43,44}$ Parameters of the artificially created roughness in experiment ${ }^{43}$ were $l, R \sim 10^{-2} \mathrm{~cm}$. Experimentally, the velocity distribution around the average value of $v$ is very narrow and the fraction of low-velocity neutrons is insignificant. Thus, $H \gg l, R \gg L$ meaning that $S \gg 1$ and that the quasiclassical expression in Eq. (71) provides a fairly accurate description of diffusion and relaxation parameters.

A similar system is the system of electrons above helium or hydrogen surface in weak electric field. Numerically, in fields $\mathcal{E}=10^{3} \mathrm{~V} / \mathrm{cm}, \quad m g$ in the above equations should be replaced by $e \mathcal{E}=1.6 \times 10^{-9} \mathrm{erg} / \mathrm{cm}$, while $L=\left(\pi^{2} / \mathrm{me} \mathcal{E}\right)^{1 / 3}$ $=3.78 \times 10^{-6} \mathrm{~cm}$. The electron-helium system ${ }^{45,46}$ differs from the trapped ultracold neutrons in two ways. First, the inhomogeneities of the surface of liquid helium or thick helium films are ripplons (at $T \sim 1 \mathrm{~K}, \quad R \sim 20 \AA, \quad l$ $\sim 0.8 \AA$ ) and are not static. Though this does not necessarily change the results, a more direct application is the electron system above a thin helium film on the surface of inhomogeneous solid substrate in a setup similar to the quasi-1D electron-helium system of Ref. 47 or electrons on solid hydrogen. The scale of inhomogeneities in a setup of the type ${ }^{47}$ is large, $l \sim R \sim 1 \mu m \gg L$ meaning that $S \gg 1$ and that one should use the quasiclassical results for diffusion coefficient. Another peculiarity is that the electron in strong electric field creates a dimple on the helium surface. This makes the effective mass dependent on the electric field and leads, in the limit of large fields, to selftrapping or auto-localization of electrons in heavy ripplonic polarons. As a result, our description can be used without modifications only in the relatively low electric fields. The most promising application of this type may be the system of electrons on the surface of solid hydrogen.

\section{Scattering by surface inhomogeneities and localization}

The above expressions for diffusion coefficient $D(E)$ and mean-free path $\mathcal{L}=2 \mathrm{D} / \mathrm{v}$ determine the (weak) localization length $\mathcal{R}$ for particles with energy $E$ (Refs. 14 and 8)

$$
\mathcal{R}(E)=\mathcal{L}(E) \exp [\varphi(E)], \quad \varphi(E)=\pi m S(E) D(E)
$$

where $S$ is the number of minibands $\epsilon_{j \mathbf{q}}$ accessible for a particle with energy $E$.

The diffusion results from the previous sections provide an adequate description of the localization exponent $\varphi$ (75) in various systems. Experimental observation of the weak 2D localization (75) is possible when the exponent $\varphi$ is not very large, $\varphi \leqq 20$. In order to have a reasonable localization length, one should try to decrease the particle energy $E$, decrease the correlation radius $R$ and the thickness $L$, and increase the amplitude of inhomogeneities $l$. Numerical estimates show that the 2D localization (75) can be observed almost exclusively for low-energy particles for which only the first miniband $\epsilon_{1 \mathrm{q}}$ is accessible, $S(E)=1$. At higher energies, the exponent (75) becomes too large.

Comparison of Eqs. (46), (65), and (69) for diffusion of the low-energy particles within the single miniband shows that the localization exponent can be written in a universal form as

$$
\varphi(E)=\frac{\pi^{2}}{2 R^{4} l^{2}}\left(\frac{L}{\pi}\right)^{6} \frac{\Phi(q R)}{a_{11}+a_{22}+2 a_{12}}
$$

[in single-wall systems, the factor $1 /\left(a_{11}+a_{22}+2 a_{12}\right)$ should be replaced by 1]. The plot of function $\Phi(x)$, Eq. (46), is given in Fig. 9. This function grows very rapidly with increasing $x$, and one is unlikely to see localization for $q R$ $>1.5$. The universality of the coefficient in Eq. (76) for all these diverse systems is explained by the choice of coefficients in the definition of the spatial scale $L$ via the derivative of the normalized wave function on the wall (63).

After $q R$ reaches a certain critical value $x_{c}=q_{c} R$, the second energy miniband becomes accessible and the localization exponent $\varphi$ is not described by Eq. (76) and Fig. 9 any more. These critical values $x_{c}$ are different for different systems. For example, for channels with two impenetrable walls $x_{c}=q_{c} R=\sqrt{3} \pi R / L$; for a bouncing ball, the critical value is smaller, $\quad x_{c}=q_{c} R \equiv 2^{1 / 3} \pi^{2 / 3}\left(\tilde{\epsilon}_{2}-\tilde{\epsilon}_{1}\right)^{1 / 2}(R / L)=3.57 R / L$. These critical values of $x_{c}=q_{c} R$ are outside Fig. 9 for all $L \leqslant R$. The localization with more than one accessible minibands can be observed only for $R / L \ll 1$. The multiband lo- 
calization exponent is not universal because the spectra and interband gaps depend on the system geometry though the typical energy dependence of the localization exponent resembles the sawlike curve in Fig. 6.

\section{DISCUSSION AND SUMMARY}

In summary, we derived general expressions for the relaxation time operator for ballistic particles scattered by weak surface roughness in quantized systems. These expressions provide, often analytically, the values of transport and localization parameters. The results are illustrated for such diverse systems as classical or degenerate particles in ultrathin films and channels, multilayer systems, single-particle diffusion, diffusion of particles weakly bound to rough substrates, quantum bouncing balls with rough walls, etc.

Outside of the quantum resonance region, the transport equation and relaxation time operator (29), expressions (24)-(27) for the transition probabilities via the intra- and interwall correlation functions of surface corrugation, and the analytical expressions (30)-(32) for the corrugationinduced relaxation time, are system independent. All systemspecific information is hidden in the energy spectrum $\boldsymbol{\epsilon}_{j}(\mathbf{q})$ and the values of unperturbed wave functions $\Psi\left(x_{\alpha}\right)$ (or their derivatives) on the walls. Inside the resonance region described in Ref. 6, the situation is much more complicated even numerically. At present, we cannot provide any general expressions, including those for effective scattering probabilities, for this regime and can approach the problem only on a system-by-system basis. Needless to say, all the difficulties associated with the resonance region disappear in the quasiclassical regime, i.e., for large-scale systems.

Most of the problems discussed in the paper are either transport problems or transport-related ones. The natural question is whether it is possible to get a similar description for other physical problems such as the effect of weak sur- face roughness on particle spectra, mean field, diffraction patterns, etc. In short, the answer is no. A simple picture exists only for the effects that can be described with the help of collision integrals with the energy $\delta$ functions $\delta\left(E_{1}\right.$ $\left.-E_{2}\right)$ in them (2). The wall-induced spectral changes involve parts of the collision operator not with the energy $\delta$ functions $\delta\left(E_{1}-E_{2}\right)$, but with the principal part integrals $P\left(1 / E_{1}-E_{2}\right)$. As a result, such processes should take into account the terms $\left(E_{1}-E_{2}\right)\left\langle\delta \psi_{1} \mid \psi_{2}^{(0)}\right\rangle$ in the matrix elements (4). These terms, in turn, are not universal and cannot be easily incorporated into the equations.

Similar argument explains the difficulty of calculations in the quantum resonance regime. In this regime, there is a strong coupling between pure and mixed quantum states of particles (coupling between diagonal and off-diagonal Green's functions) that requires to include the principal part integrals along with the energy $\delta$ functions even in the parts of the collision operator responsible for transport. Even intuitively, it is clear that in resonance the matrix elements $\left(E_{1}\right.$ $\left.-E_{2}\right)\left\langle\delta \psi_{1} \mid \psi_{2}^{(0)}\right\rangle$ do not disappear from the equations. We want to emphasize that all these difficulties associated with the resonance regime exist for quantized films with usual bulk impurities as well. As it was shown in Ref. 6, the quantum resonance regime $\Omega_{j j^{\prime}} \hat{\tau} \sim 1$ corresponds to a moderately large number $S$ of accessible minibands $\epsilon_{j \mathbf{q}}$. The quasiclassical domain of large quantum numbers $S \gg 1$ (thick films) and the ultraquantum case of small $S$ (ultrathin films) are outside of the anomalous resonance region.

The next step should be the simultaneous study of boundary and bulk scattering in thin films including the interference terms beyond the Matthiessen's rule.

\section{ACKNOWLEDGMENT}

This work was supported by NSF Grant No. DMR9705304.
${ }^{1}$ P. Beckmann and A. Spizzichino, The Scattering of Electromagnetic Waves from Random Surfaces (Pergamon, New York, 1963).

${ }^{2}$ F. G. Bass and I. M. Fuks, Wave Scattering from Statistically Rough Surfaces (Pergamon, New York, 1979).

${ }^{3}$ Interactions of Atoms and Molecules with Solid Surfaces, edited by V. Bortolani, N. H. March, and M. P. Tosi (Plenum, New York, 1990).

${ }^{4}$ J. A. Ogilvy, Theory of Wave Scattering from Random Surfaces (Hilger, Bristol, 1991).

${ }^{5}$ A. G. Voronovich, Wave Scattering at Rough Surfaces (Springer, Berlin, 1994).

${ }^{6}$ A. E. Meyerovich and A. Stepaniants, Phys. Rev. B 58, 13242 (1998).

${ }^{7}$ J. Rammer and H. Smith, Rev. Mod. Phys. 58, 323 (1986).

${ }^{8}$ A. McGurn and A. Maradudin, Phys. Rev. B 30, 3136 (1984).

${ }^{9}$ P. Arseyev, Pis'ma Zh. Éksp. Teor. Fiz. 45, 132 (1987) [JETP Lett. 45, 163 (1987)]

${ }^{10}$ L. I. Glazman, G. B. Lesovik, D. E. Khmel'nitskii, and R. I. Shekhter, Pis'ma Zh. Éksp. Teor. Fiz. 48, 218 (1988) [JETP Lett. 48, 238 (1988)].
${ }^{11}$ D. A. Wharam, T. J. Thornton, R. Newbury, M. Pepper, M. Ahmed, J. E. F. Frost, D. G. Masko, D. C. Peacock, D. A. Ritchie, and G. A. C. Jones, J. Phys. C 21, L209 (1988).

${ }^{12}$ B. J. van Wees, H. van Houten, C. W. J. Beenakker, J. G. Williamson, L. P. Kouwenhoven, D. van der Marel, and C. T. Foxon, Phys. Rev. Lett. 60, 848 (1988).

${ }^{13}$ V. I. Kozub and A. A. Krokhin, J. Phys.: Condens. Matter 5, 9135 (1993).

${ }^{14}$ P. A. Lee and T. V. Ramakrishnan, Rev. Mod. Phys. 57, 287 (1985).

${ }^{15}$ B. Altshuler, in Nanostructures and Mesoscopic Systems, edited by W. P. Kirk and M. A. Reed (Academic Press, New York, 1991), pp. 405-416.

${ }^{16}$ C. Itzykson and J.-M. Drouffe, Statistical Field Theory (Cambridge University Press, Cambridge, 1991), Vol. 2, Chap. 10.

${ }^{17}$ A. G. Voronovich, Akust. Zh. 30, 747 (1984) [Sov. Phys. Acoust. 30, 444 (1984)]; Zh. Éksp. Teor. Fiz. 89, 116 (1985) [Sov. Phys. JETP 62, 65 (1985)].

${ }^{18}$ N. M. Makarov, A. V. Moroz, and V. A. Yampol'skii, Phys. Rev. B 52, 6087 (1995).

${ }^{19}$ A. Kawabata, J. Phys. Soc. Jpn. 62, 3988 (1993). 
${ }^{20}$ Z. Tesanovic, M. V. Jaric, and S. Maekawa, Phys. Rev. Lett. 57, 2760 (1986).

${ }^{21}$ N. Trivedi and N. W. Ashcroft, Phys. Rev. B 38, 12298 (1988).

${ }^{22}$ A. E. Meyerovich and S. Stepaniants, Phys. Rev. Lett. 73, 316 (1994).

${ }^{23}$ A. E. Meyerovich and S. Stepaniants, Phys. Rev. B 51, 17116 (1995).

${ }^{24}$ A. E. Meyerovich and S. Stepaniants, J. Phys.: Condens. Matter 9, 4157 (1997).

${ }^{25}$ A. M. Bratkovsky and S. N. Rashkeev, Phys. Rev. B 53, 13074 (1996).

${ }^{26}$ J. A. Konrady, Jr., J. Acoust. Soc. Am. 56, 1687 (1974).

${ }^{27}$ F. Bass, V. D. Freulicher, and I. Fuks, IEEE Trans. Antennas Propag. 22, 278 (974); 22, 288 (1974).

${ }^{28}$ E. I. Thorsos and D. R. Jackson, J. Acoust. Soc. Am. 86, 261 (1989)

${ }^{29}$ H. D. I. Abrabanel, J. Acoust. Soc. Am. 68, 1459 (1980).

${ }^{30}$ J. G. Watson and J. B. Keller, J. Acoust. Soc. Am. 75, 1705 (1984).

${ }^{31}$ E. I. Thorsos and D. R. Jackson, J. Acoust. Soc. Am. 86, 261 (1989).

${ }^{32}$ R. E. Goldstein, A. I. Pesci, and V. Romero-Rochin, Phys. Rev. A 41, 5504 (1990).

${ }^{33}$ A. B. Isers, A. A. Puzenko, and I. M. Fuks, J. Electromagn. Waves Appl. 5, 1419 (1991).
${ }^{34}$ D. H. Berman, J. Acoust. Soc. Am. 92, 309 (1992); 96, 417 (1994).

${ }^{35}$ G. Fishman and D. Calecki, Phys. Rev. Lett. 62, 1302 (1989); Phys. Rev. B 43, 11581 (1991).

${ }^{36}$ G. Palasantzas and J. Barnas, Phys. Rev. B 56, 7726 (1997).

${ }^{37}$ I. B. Altfeder, D. M. Chen, and K. A. Matveev, Phys. Rev. Lett. 80, 4895 (1998).

${ }^{38}$ R. M. Feenstra et al., Phys. Rev. Lett. 72, 2749 (1994).

${ }^{39}$ I. Silvera, J. Low Temp. Phys. 101, 49 (1995).

${ }^{40}$ J. T. M. Walraven, in Fundamental Systems in Quantum Optics, edited by J. Dalibard, J. M. Raimond, and J. Zinn-Justin (Elsevier, New York, 1992), p. 485.

${ }^{41}$ B. Lushchikov and A. I. Frank, Pis'ma Zh. Éksp. Teor. Fiz. 28, 607 (1975) [JETP Lett. 28, 559 (1978)].

${ }^{42}$ A. Stepaniants, D. Sarkisov, A. Steyerl, and A. Meyerovich, J. Low Temp. Phys. 113, 1159 (1998).

${ }^{43}$ P. Geltenbort, S. Malik, and A. Steyerl (unpublished).

${ }^{44}$ V. P. Alfimenkov et al., Pis'ma Zh. Éksp. Teor. Fiz. 55, 92 (1992) [JETP Lett. 55, 84 (1992)].

${ }^{45}$ A. J. Dahm, Low Temp. Phys. 23, 639 (1997); I. Karakurt and A. Dahm, J. Low Temp. Phys. 113, 1091 (1998).

${ }^{46}$ S. S. Sokolov, G.-Q. Hai, and N. Studart, Phys. Rev. B 55, R3370 (1977).

${ }^{47}$ Yu. Z. Kovdrya, V. A. Nikolaenko, H. Yayama, A. Tomokiyo, O. I. Kirichek, and I. B. Berkutov, J. Low Temp. Phys. 110, 191 (1998). 